\title{
The Engulfing Property for Sections of Convex Functions on the Heisenberg Group and the Associated Quasi-distance
}

\author{
A. Calogero ${ }^{1}\left(\right.$ C) $\cdot$ R. Pini ${ }^{1}$
}

Received: 31 July 2020 / Accepted: 28 February 2021 / Published online: 5 April 2021

(C) The Author(s) 2021

\begin{abstract}
In this paper we investigate the property of engulfing for $H$-convex functions defined on the Heisenberg group $\mathbb{H}^{n}$. Starting from the horizontal sections introduced by Capogna and Maldonado (Proc Am Math Soc 134:3191-3199, 2006), we consider a new notion of section, called $\mathbb{H}^{n}$-section, as well as a new condition of engulfing associated to the $\mathbb{H}^{n}$-sections, for an $H$-convex function defined in $\mathbb{H}^{n}$. These sections, that arise as suitable unions of horizontal sections, are dimensionally larger; as a matter of fact, the $\mathbb{H}^{n}$-sections, with their engulfing property, will lead to the definition of a quasi-distance in $\mathbb{H}^{n}$ in a way similar to Aimar et al. in the Euclidean case ( $\mathrm{J}$ Fourier Anal Appl 4:377-381, 1998). A key role is played by the property of round $H$-sections for an $H$-convex function, and by its connection with the engulfing properties.
\end{abstract}

Keywords Heisenberg group $\cdot H$-convex function - Section of $H$-convex function Engulfing property $\cdot$ Quasi-distance $\cdot$ Round $H$-sections

Mathematics Subject Classification $52 \mathrm{~A} 30 \cdot 26 \mathrm{~A} 12 \cdot 26 \mathrm{~B} 25$

\section{Introduction}

Given a convex function $u: \mathbb{R}^{n} \rightarrow \mathbb{R}$, for every $x_{0} \in \mathbb{R}^{n}, p \in \partial u\left(x_{0}\right)$, and $s>0$, we will denote by $S_{u}\left(x_{0}, p, s\right)$ the section of $u$ at $x_{0}$ with height $s$, defined as follows

$$
S_{u}\left(x_{0}, p, s\right)=\left\{x \in \mathbb{R}^{n}: u(x)-u\left(x_{0}\right)-p \cdot\left(x-x_{0}\right)<s\right\}
$$

$\triangle$ R. Pini

rita.pini@unimib.it

A. Calogero

andrea.calogero@unimib.it

1 Dipartimento di Matematica e Applicazioni, Università degli Studi di Milano-Bicocca, Via R. Cozzi 55, 20125 Milan, Italy 
in case $u$ is differentiable at $x_{0}$, we will denote the section by $S_{u}\left(x_{0}, s\right)$, for short. The related notion of engulfing for convex functions, or, equivalently, for their sections, is essentially a geometric property, and it is based on a regular mutual behaviour of the sections of the function. We say that a convex function $u$ satisfies the engulfing property (shortly, $u \in E\left(\mathbb{R}^{n}, K\right)$ ) if there exists $K>1$ such that for any $x \in \mathbb{R}^{n}$, $p \in \partial u(x)$, and $s>0$, if $y \in S_{u}(x, p, s)$, then $S_{u}(x, p, s) \subset S_{u}(y, q, K s)$, for every $q \in \partial u(y)$.

The functions $u$ in the class $E\left(\mathbb{R}^{n}, K\right)$ have been studied in connection with the solution to the Monge-Ampère equation det $D^{2} u=\mu$, where $\mu$ is a Borel measure on $\mathbb{R}^{n}$. In this framework, a $\mathcal{C}^{1, \beta}$-estimate for the strictly convex, generalized solutions to the Monge-Ampère equation was proved by Caffarelli $([7,8])$, under the assumption that the measure $\mu$ satisfies a suitable doubling property (see the exhaustive book by Gutiérrez [19]). This doubling property is actually equivalent to the geometric property of engulfing for the solution $u$.

Another issue is related to the properties enjoyed by the family of sections $\left\{S_{u}(x, s)\right\}_{\left\{x \in \mathbb{R}^{n}, s>0\right\}}$, in case $u$ is a convex differentiable function in $E\left(\mathbb{R}^{n}, K\right)$. In [1], it is shown that, in this case, one can define a quasi-distance $d$ on $\mathbb{R}^{n}$ as follows:

$$
d(x, y):=\inf \left\{s>0: x \in S_{u}(y, s), y \in S_{u}(x, s)\right\} .
$$

In addition, if $B_{d}(x, r)$ is a $d$-ball of center $x$ and radius $r$, then

$$
S_{u}\left(x, \frac{r}{2 K}\right) \subset B_{d}(x, r) \subset S_{u}(x, r) .
$$

In the archetypal case $u(x)=\|x\|^{2}$, with $x \in \mathbb{R}^{n}$, one has $S_{u}(x, s)=B^{\mathbb{R}^{n}}(x, \sqrt{s})$, and hence the family of sections of $u$ consists of the usual balls in $\mathbb{R}^{n}$.

In the case of convex functions defined in a Carnot group $\mathbf{G}$, in [13] Capogna and Maldonado introduced some appropriate geometric objects, that can be considered as the sub-Riemmannian analogue of the classical sections, as well as a naturally related notion of horizontal engulfing. Given a horizontally convex function $\varphi: \mathbf{G} \rightarrow \mathbb{R}$, $\xi_{0} \in \mathbf{G}, p \in \mathbb{R}^{m_{1}}, s>0$, the section $S_{u}^{H}\left(\xi_{0}, p, s\right)(H$-sections, from now on, where $H$ stands for horizontal) is defined as follows:

$$
S_{\varphi}^{H}\left(\xi_{0}, p, s\right):=\left\{\xi_{0} \circ \exp v: v \in V_{1}, \varphi\left(\xi_{0} \circ \exp v\right)-\varphi\left(\xi_{0}\right)-v \cdot p<s\right\}
$$

where $V_{1} \cong \mathbb{R}^{m_{1}}$ is the first layer of the stratification of the Lie algebra of $\mathbf{G}$; in case $\varphi$ is horizontally differentiable at $\xi_{0}$, we will denote such $H$-section by $S_{\varphi}^{H}\left(\xi_{0}, s\right)$, for short. The mentioned authors say that a horizontally convex and differentiable function $\varphi$ satisfies the engulfing property if there exists $K>1$ such that, for every $\xi$, $\xi^{\prime} \in \mathbf{G}$ and $s>0$, if $\xi^{\prime} \in S_{\varphi}^{H}(\xi, s)$, then $\xi \in S_{\varphi}^{H}\left(\xi^{\prime}, K s\right)$. Let us stress that the definition of $H$-section in (1.4) and the notion of engulfing are affected by the subRiemannian structure exactly as the notion of horizontal convexity; more precisely, they rely upon the behaviour of the function on the horizontal lines and planes. In [13] it is proved that a strictly convex and everywhere differentiable function on a Carnot group, satisfying this horizontal version of engulfing, belong to the Folland-Stein class 
$\Gamma^{1+1 / K}$, i.e., the horizontal derivatives $X_{i} \varphi$ are $1 / K$-Hölder continuous with respect to any left-invariant and homogeneous pseudo-norm in the group. The key point in their argument is a reduction of the general discussion to the one-dimensional case. As a matter of fact, the "dimension" of the $H$-sections in (1.4) is the dimension of the first layer of the stratification of the Lie algebra of the group; in particular, these $H$-sections have empty interior. This fact prevents from building a quasi-distance as in (1.2) starting from the family of sections associated to every point of the group.

In this paper we focus on horizontally convex functions $\varphi$ ( $H$-convex functions) on the Heisenberg group $\mathbb{H}^{n}$, that is the simplest Carnot group of step 2. Our main purpose is to overcome the dimensional gap between the $H$-sections, defined in [13], and the balls related to any quasi-distance in $\mathbb{H}^{n}$, by introducing and studying a different notion of section. Our idea takes inspiration from the notion of $H$-section in (1.4), together with the property that any pair of points in $\mathbb{H}^{n}$ can be joined by at most three consecutive horizontal segments. These facts lead us to define full-dimensional sections that arise as a sort of composition in three steps of "thin" $H$-sections. These new objects will be called $\mathbb{H}^{n}$-section, and will be denoted by $\mathbb{S}_{\varphi}^{\mathbb{H}^{n}}\left(\xi_{0}, p, s\right)$ (for the precise definition of $\mathbb{S}_{\varphi}^{\mathbb{H}^{n}}\left(\xi_{0}, p, s\right)$, see Definition 5.1). For these $\mathbb{H}^{n}$-sections, we introduce the following engulfing condition:

Definition 1.1 Let $\varphi: \mathbb{H}^{n} \rightarrow \mathbb{R}$ be an $H$-convex function. We say that $\varphi$ satisfies the engulfing property $E\left(\mathbb{H}^{n}, K\right)$ if there exists $K>1$ such that for any $\xi \in \mathbb{H}^{n}, p \in$ $\partial_{H} \varphi(\xi)$ and $s>0$, if $\xi^{\prime} \in \mathbb{S}_{\varphi}^{\mathbb{H}^{n}}(\xi, p, s)$, then

$$
\mathbb{S}_{\varphi}^{\mathbb{H}^{n}}(\xi, p, s) \subset \mathbb{S}_{\varphi}^{\mathbb{H}^{n}}\left(\xi^{\prime}, q, K s\right)
$$

for every $q \in \partial_{H} \varphi\left(\xi^{\prime}\right)$.

It is obvious that a function which satisfies this engulfing property $E\left(\mathbb{H}^{n}, K\right)$, satisfies the engulfing property introduced by Capogna and Maldonado.

The study of this new notion of engulfing for $\mathbb{H}^{n}$-sections of full dimension requires a mix of tools and properties inherited by the Euclidean case $\mathbb{R}^{n}$, both for the simplest case $n=1$, and for the knotty case $n>1$. Following the idea in [21] and, in particular, the equivalence between iii. and iv in Theorem 7.1 below, we introduce and study a horizontal notion of round sections for the $H$-sections (see Definition 3.1). We prove that every $H$-convex function with round $H$-sections satisfies the engulfing property $E\left(\mathbb{H}^{n}, K\right)$ in Definition 1.1.

Let us summarize our results as follows:

Theorem 1.1 Let $\varphi: \mathbb{H}^{n} \rightarrow \mathbb{R}$ be an $H$-convex function with round $H$-sections, then

i. $\varphi$ satisfies the engulfing property $E\left(\mathbb{H}^{n}, K\right)$; consequently, in the class of $H$-convex functions with round $H$-sections, the engulfing for $H$-sections and the engulfing for $\mathbb{H}^{n}$-sections are equivalent properties;

ii. the function $d_{\varphi}: \mathbb{H}^{n} \times \mathbb{H}^{n} \rightarrow[0,+\infty)$ defined by

$$
d_{\varphi}\left(\xi, \xi^{\prime}\right)=\inf \left\{s>0: \xi \in \mathbb{S}_{\varphi}^{\mathbb{H}^{n}}\left(\xi^{\prime}, s\right), \xi^{\prime} \in \mathbb{S}_{\varphi}^{\mathbb{H}^{n}}(\xi, s)\right\}
$$


is a quasi-distance in $\mathbb{H}^{n}$; moreover, for the $d_{\varphi}$-balls, an $\mathbb{H}^{n}$-version of the inclusions in (1.3) holds true (see (6.10) below).

Here, the archetypal example in $\mathbb{H}$ of the $H$-convex function $\varphi(x, y, t)=x^{2}+y^{2}$ gives $S_{\varphi}^{\mathbb{H}}(\xi, s)=\widetilde{B}(\xi, \sqrt{s})$, that is, the family of $\mathbb{H}$-sections of $\varphi$ consists of the $\widetilde{B}$-balls of a left-invariant and homogeneous distance $\widetilde{d}$ (see (5.2) and Example 6.1).

The property of round $H$-sections is actually stronger than the horizontal engulfing; we are able to provide an example of an $H$-convex function which satisfies the horizontal engulfing property but does not have round $H$-sections, and this phenomenon appears also in the Euclidean case, if $n>1$. Nevertheless, the main issue of the result above relies upon the dimensional gap between the assumptions, where a purely horizontal property is required, and the final result, where full-dimensional sets are involved.

The paper is organized as follows. In Sect. 2 we recall some results related to the engulfing property for a function defined in $\mathbb{R}^{n}$, together with the structure of $\mathbb{H}^{n}$ and the notion of horizontal convexity. In Sect. 3 we introduce the $H$-sections, and we show that round $H$-sections and controlled $H$-slope are equivalent property for these $H$-sections (see Theorem 3.1). In Sect. 4 we characterize the functions with the engulfing property $E(H, K)$, and prove that the two properties introduced in the previous section are sufficient conditions for a function to be in $E(H, K)$. In Sect. 5 we move to the notion of $\mathbb{H}^{n}$-sections and the related engulfing property as in Definition 1.1, and we prove Theorem 1.1 i. In Sect. 6 we prove Theorem 1.1 ii. and provide a concrete example. In the final section we list some open questions.

\section{Preliminary Notions and Results}

In the paper, we will deal with $H$-convex functions defined on the Heisenberg group $\mathbb{H}^{n}$. As we will see later, the notion of $H$-convexity requires that, for every point $\xi \in \mathbb{H}^{n}$, one looks at the behaviour of the function under two points of view. The first one is one-dimensional, since the restriction of the function to any horizontal line $\{\xi \circ \exp t v\}_{t \in \mathbb{R}}$, with $v \in V_{1}$, is an ordinary convex function; the second one is $2 n$ dimensional, according to the fact that $v \in V_{1} \cong \mathbb{R}^{2 n}$, or, equivalently, the horizontal lines through $\xi$ span the $2 n$-dimensional horizontal plane $H_{\xi}$. For these reasons, the first part of this section will be devoted to some results related to the engulfing property of convex functions $u: \mathbb{R}^{n} \rightarrow \mathbb{R}$, both in the case $n=1$, and in the case $n \geq 2$. In the second part we will recall the notion of $H$-convexity, together with some related results, for functions defined on the Heisenberg group $\mathbb{H}^{n}$.

\subsection{The Engulfing Property for Convex Functions in $\mathbb{R}^{n}$}

Let us concentrate, first, on the one-dimensional case, i.e. $n=1$. The following characterization holds (see Theorem 2 in [18], Theorem 5.1 in [14]):

Theorem 2.1 Let $u: \mathbb{R} \rightarrow \mathbb{R}$ be a strictly convex and differentiable function. The following are equivalent: 
i. $u \in E(\mathbb{R}, K)$, for some $K>1$;

ii. there exists a constant $K^{\prime}>1$ such that, if $x, y \in \mathbb{R}$ and $s>0$ verify $x \in S_{u}(y, s)$, then $y \in S_{u}\left(x, K^{\prime} s\right)$;

iii. there exists a constant $K^{\prime \prime}>1$ such that, for any $x, y \in \mathbb{R}$,

$$
\begin{aligned}
& \frac{K^{\prime \prime}+1}{K^{\prime \prime}}\left(u(y)-u(x)-u^{\prime}(x)(y-x)\right) \leq\left(u^{\prime}(x)-u^{\prime}(y)\right)(x-y) \\
& \leq\left(K^{\prime \prime}+1\right)\left(u(y)-u(x)-u^{\prime}(x)(y-x)\right) .
\end{aligned}
$$

As a matter of fact, the assumption of differentiability in the theorem above can be removed, as proved in [11]:

Theorem 2.2 Let $u: \mathbb{R} \rightarrow \mathbb{R}$ be a convex function, with bounded sections, satisfying the engulfing property. Then, $u$ is strictly convex and is in $\mathcal{C}^{1}(\mathbb{R})$.

Given a strictly convex differentiable function $u: \mathbb{R} \rightarrow \mathbb{R}$, one can consider the associated Monge-Ampère measure $\mu_{u}$ defined on any Borel set $A \subset \mathbb{R}$ by

$$
\mu_{u}(A)=\left|u^{\prime}(A)\right|
$$

where $|\cdot|$ denotes the Lebesgue measure. We say that the measure $\mu_{u}$ has the $(D C)$ doubling property if there exist constants $\alpha \in(0,1)$ and $C>1$ such that

$$
\mu_{u}\left(S_{u}(x, s)\right) \leq C \mu_{u}\left(\alpha S_{u}(x, s)\right)
$$

for every section $S_{u}(x, s)$ (here $\alpha S_{u}(x, s)$ is the open convex set obtained by $\alpha$ contraction of $S_{u}(x, s)$ with respect to its center of mass). In [20] and [17] it was shown that the (DC)-doubling property of the measure $\mu_{u}$ is equivalent to the engulfing property for the function $u$; in particular, given $u$ in $E(\mathbb{R}, K)$, the constants $\alpha$ and $C$ in (2.2) depend only on $K$. A Radon measure $\mu$ is doubling if and only if there exists a constant $A$ such that

$$
\frac{1}{A} \leq \frac{\mu\left(Q_{1}\right)}{\mu\left(Q_{2}\right)} \leq A,
$$

for any congruent cubes $Q_{1}$ and $Q_{2}$ with nonempty intersection (see, for example, [22]). We recall that two subsets of $\mathbb{R}$ are called congruent if there exists an isometry of $\mathbb{R}$ that maps one of them onto the other. Since every open and bounded interval in $\mathbb{R}$ is a particular section for $u$, the (DC)-doubling property of $\mu_{u}$ is trivially equivalent to the fact that $\mu_{u}$ is a doubling measure. In particular, the constant $A$ depends only on $K$. Now, noticing that $\mu_{u}((x, x+r))=u^{\prime}(x+r)-u^{\prime}(x)$, by (2.1) we obtain

$$
\begin{aligned}
& \frac{K^{\prime \prime}+1}{K^{\prime \prime}}\left(u(x+r)-u(x)-u^{\prime}(x) r\right) \\
& \left.\quad \leq r \mu_{u}((x, x+r))\right) \leq\left(K^{\prime \prime}+1\right)\left(u(x+r)-u(x)-u^{\prime}(x) r\right) .
\end{aligned}
$$

These arguments show the central role of the function $(x, r) \mapsto u(x+r)-u(x)-u^{\prime}(x) r$ in our paper. More precisely in [14] (see Theorem 5.5) the authors prove the following: 
Theorem 2.3 Let $u: \mathbb{R} \rightarrow \mathbb{R}$ be a strictly convex and differentiable function. Then $u \in E(\mathbb{R}, K)$ if and only if there exist two constants $A_{1}>1$ and $A_{2}>1$, both of them depending on $K$, such that

$$
\begin{aligned}
& \frac{1}{A_{1}} \leq \frac{u(x+r)-u(x)-u^{\prime}(x) r}{u(x-r)-u(x)+u^{\prime}(x) r} \leq A_{1}, \quad \forall x \in \mathbb{R}, r>0 ; \\
& \frac{1}{A_{2}} \leq \frac{u(x+2 r)-u(x)-u^{\prime}(x) 2 r}{u(x+r)-u(x)-u^{\prime}(x) r} \leq A_{2}, \quad \forall x \in \mathbb{R}, r>0 .
\end{aligned}
$$

Condition (2.4) says that $u$ is essentially symmetric around every point, and condition (2.5) says that it satisfies the so-called $\Delta_{2}$ condition at each point in $\mathbb{R}$.

Hence, the behaviour of the measure $\mu_{u}$ is related to the functions $m_{u}, M_{u}$ : $\mathbb{R} \times \mathbb{R}^{+} \rightarrow \mathbb{R}^{+}$defined by

$$
\begin{aligned}
& m_{u}(x, r):=\min _{\{z:|z-x|=r\}}\left(u(z)-u(x)-u^{\prime}(x)(z-x)\right) \\
& M_{u}(x, r):=\max _{\{z:|z-x|=r\}}\left(u(z)-u(x)-u^{\prime}(x)(z-x)\right),
\end{aligned}
$$

for every $x \in \mathbb{R}, r \in \mathbb{R}^{+}$. These functions will be naturally extended to the $n$ dimensional case and in $\mathbb{H}^{n}$, and will play a crucial role in the investigation of the engulfing for $H$-convex functions.

For every fixed $x \in \mathbb{R}$, denote by $u_{x}$ the function

$$
s \mapsto u_{x}(s)=u(x+s)-u(x)-u^{\prime}(x) s .
$$

Then, $M_{u}(x, r) \in\left\{u_{x}( \pm r)\right\}$, and $M_{u}(x, 2 r) \in\left\{u_{x}( \pm 2 r)\right\}$. Let us suppose, for instance, that the following equalities hold true:

$$
\begin{aligned}
& M_{u}(x, 2 r)=u_{x}(2 r), \quad m_{u}(x, 2 r)=u_{x}(-2 r), \\
& M_{u}(x, r)=u_{x}(r), \quad m_{u}(x, r)=u_{x}(-r) .
\end{aligned}
$$

Then, by (2.4) and (2.5), we obtain

$$
\begin{aligned}
& M_{u}(x, 2 r)=u_{x}(2 r) \leq A_{2} u_{x}(r)=A_{2} M_{u}(x, r), \\
& m_{u}(x, 2 r)=u_{x}(-2 r) \leq A_{1} u_{x}(2 r) \leq A_{1} A_{2} u_{x}(r) \leq A_{1}^{2} A_{2} u_{x}(-r)=A_{1}^{2} A_{2} m_{u}(x, r), \\
& M_{u}(x, r)=u_{x}(r) \leq A_{1} u_{x}(-r)=A_{1} m_{u}(x, r) .
\end{aligned}
$$

The other possible combinations can be treated similarly, and we obtain the following fundamental estimates:

Remark 2.1 Let $u \in E(\mathbb{R}, K)$ be a strictly convex and differentiable function. Then,

$$
\begin{array}{rlrl}
M_{u}(x, 2 r) & \leq B_{1} M_{u}(x, r), & & \forall x \in \mathbb{R}, r \geq 0 \\
m_{u}(x, 2 r) \leq B_{2} m_{u}(x, r), & & \forall x \in \mathbb{R}, r \geq 0 \\
M_{u}(x, r) \leq B_{3} m_{u}(x, r), & & \forall x \in \mathbb{R}, r \geq 0
\end{array}
$$


where $B_{1}, B_{2}$ and $B_{3}$ depend only on $K$ (and $B_{i}>1$ ).

It is worthwhile to note that inequality (2.10) is false if $n \geq 2$, despite the engulfing property holds; the function in (4.9), due to Wang, will provide a counterexample to this phenomenon.

The next result provides another estimate for the function $m_{u}$ :

Proposition 2.1 Let $u \in E(\mathbb{R}, K)$ be a convex function with bounded sections. Then,

$$
B_{4} m_{u}(x, r) \leq m_{u}(x, 2 r), \quad \forall x \in \mathbb{R}, r \geq 0,
$$

with $B_{4}>1$ which depends only on $K$.

Proof Let us fix $x \in \mathbb{R}$. The function $u_{x}$ defined in (2.7) is strictly convex and differentiable (see [11]), and belongs to $E(\mathbb{R}, K)$; moreover,

$$
\frac{K^{\prime \prime}+1}{K^{\prime \prime}} u_{x}(y) \leq u_{x}^{\prime}(y) y, \quad \forall y \in \mathbb{R},
$$

where $K^{\prime \prime}$ depends only on $K$ (for all the details, see Theorem 4 and its proof in [18]). Hence, for every fixed $r>0$, the Gronwall inequality gives

$$
u_{x}(|y|) \geq u_{x}(r)\left(\frac{|y|}{r}\right)^{\frac{K^{\prime \prime}+1}{K^{\prime \prime}}}, \quad \forall|y| \geq r .
$$

Therefore, we obtain that $u_{x}( \pm 2 r) \geq 2^{\frac{K^{\prime \prime}+1}{K^{\prime \prime}}} u_{x}( \pm r)$. Let $m_{u}(x, r)=u_{x}(r)$. Then, $B_{4} m_{u}(x, r) \leq u_{x}(2 r)$. Suppose that $B_{4} m_{u}(x, r)>u_{x}(-r)$. In this case, $u_{x}(-2 r) \geq$ $B_{4} u_{x}(-r)$, and thus $B_{4} u_{x}(-r)<B_{4} m_{u}(x, r)$, a contradiction. Then, (2.11) follows.

Let us now move to the case $n \geq 2$. Given a differentiable function $u: \mathbb{R}^{n} \rightarrow \mathbb{R}$, as in the one-dimensional case (2.6), the functions $m_{u}, M_{u}: \mathbb{R}^{n} \times \mathbb{R}^{+} \rightarrow \mathbb{R}^{+}$are defined by

$$
\begin{aligned}
m_{u}(x, r): & =\min _{\{z:\|z-x\|=r\}}(u(z)-u(x)-\nabla u(x) \cdot(z-x)) \\
M_{u}(x, r): & =\max _{\{z:\|z-x\|=r\}}(u(z)-u(x)-\nabla u(x) \cdot(z-x)),
\end{aligned}
$$

for every $x \in \mathbb{R}^{n}, r \in \mathbb{R}^{+}$.

Let us recall the following property, that will be critical when dealing with the engulfing in $\mathbb{H}^{n}$.

Definition 2.1 (see Definition 2.1 in [21]) Let $u: \mathbb{R}^{n} \rightarrow \mathbb{R}$ be a convex function. We say that $u$ has round sections if there exists a constant $\tau \in(0,1)$ with the following property: for every $x \in \mathbb{R}^{n}, p \in \partial u(x)$, and $s>0$, there is $R>0$ such that

$$
B(x, \tau R) \subset S_{u}(x, p, s) \subset B(x, R) .
$$


In [21] (see Theorem 7.1 below) it is proved that a convex function $u: \mathbb{R}^{n} \rightarrow \mathbb{R}$ has round sections if and only if $u$ is differentiable, but not affine, and has controlled slope, i.e., there exists a constant $H \geq 1$ such that

$$
M_{u}(x, r) \leq H m_{u}(x, r), \quad \forall x \in \mathbb{R}^{n}, r \geq 0
$$

This equivalence is quantitative, in the sense that the constants involved in each statement depend only on each other and $n$, but not on $u$. Furthermore, if $u: \mathbb{R}^{n} \rightarrow \mathbb{R}$ satisfies one of the two equivalent conditions above, then $u \in E\left(\mathbb{R}^{n}, K\right)$, for a suitable $K>1$ (see Theorem 3.9 in [21]). Let us finally notice that condition (2.12) is the $n$-dimensional version of condition (2.10): in the case $n \geq 2$, hence, the controlled slope for a function, or, equivalently, the property of round sections, is only a sufficient condition for a function to have the engulfing property.

\subsection{Convexity in the Heisenberg Group $\mathbb{H}^{\mathrm{n}}$}

The Heisenberg group $\mathbb{H}^{n}$ is the simplest Carnot group of step 2. We will recall some of the notions and background results used in the sequel. We will focus only on those geometric aspects that are relevant to our paper. For a general overview on the subject, we refer to [6] and [12].

The Lie algebra $\mathfrak{h}$ of $\mathbb{H}^{n}$ admits a stratification $\mathfrak{h}=V_{1} \oplus V_{2}$ with $V_{1}=$ $\operatorname{span}\left\{X_{i}, Y_{i} ; 1 \leq i \leq n\right\}$ being the first layer of the so-called horizontal vector fields, and $V_{2}=\operatorname{span}\{T\}$ being the second layer which is one-dimensional. We assume $\left[X_{i}, Y_{i}\right]=-4 T$ and the remaining commutators of basis vectors vanish. The exponential map exp $: \mathfrak{h} \rightarrow \mathbb{H}^{n}$ is defined in the usual way. By these commutator rules we obtain, using the Baker-Campbell-Hausdorff formula, that $\mathbb{H}^{n}$ can be identified with $\mathbb{R}^{n} \times \mathbb{R}^{n} \times \mathbb{R}$ endowed with the non-commutative group law given by

$$
\xi \circ \xi^{\prime}=(x, y, t) \circ\left(x^{\prime}, y^{\prime}, t^{\prime}\right)=\left(x+x^{\prime}, y+y^{\prime}, t+t^{\prime}+2\left(x^{\prime} \cdot y-x \cdot y^{\prime}\right)\right),
$$

where $x, y, x^{\prime}$ and $y^{\prime}$ are in $\mathbb{R}^{n}, t$ and $t^{\prime}$ in $\mathbb{R}$, and where ${ }^{\prime} .^{\prime}$ is the inner product in $\mathbb{R}^{n}$. Let us denote by $e$ the neutral element in $\mathbb{H}^{n}$. Transporting the basis vectors of $V_{1}$ from the origin to an arbitrary point of the group by a left-translation, we obtain a system of left-invariant vector fields written as first order differential operators as follows

$$
X_{j}=\partial_{x_{j}}+2 y_{j} \partial_{t}, \quad Y_{j}=\partial_{y_{j}}-2 x_{j} \partial_{t}, \quad j=1, \ldots, n .
$$

Via the exponential map exp $: \mathfrak{h} \rightarrow \mathbb{H}$ we identify the vector $\sum_{i=1}^{n}\left(\alpha_{i} X_{i}+\beta_{i} Y_{i}\right)+$ $\gamma T$ in $\mathfrak{h}$ with the point $\left(\alpha_{1}, \ldots, \alpha_{n}, \beta_{1}, \ldots, \beta_{n}, \gamma\right)$ in $\mathbb{H}^{n}$; the inverse $\xi: \mathbb{H}^{n} \rightarrow \mathfrak{h}$ of the exponential map has the unique decomposition $\xi=\left(\xi_{1}, \xi_{2}\right)$, with $\xi_{i}: \mathbb{H}^{n} \rightarrow V_{i}$, and we identify $V_{1}$ with $\mathbb{R}^{2 n}$ when needed.

For every positive $\lambda$, the non-isotropic Heisenberg dilation $\delta_{\lambda}: \mathbb{H}^{n} \rightarrow \mathbb{H}^{n}$ is defined by $\delta_{\lambda}(x, y, t)=\left(\lambda x, \lambda y, \lambda^{2} t\right)$. Let $N(x, y, t)=\left(\left(\|x\|^{2}+\|y\|^{2}\right)^{2}+t^{2}\right)^{\frac{1}{4}}$ be the gauge norm in $\mathbb{H}^{n}$. The function $d_{g}: \mathbb{H}^{n} \times \mathbb{H}^{n} \rightarrow[0,+\infty)$ defined by

$$
d_{g}\left(\xi, \xi^{\prime}\right):=N\left(\left(\xi^{\prime}\right)^{-1} \circ \xi\right)
$$


satisfies the triangle inequality, thereby defining a distance on $\mathbb{H}^{n}$ : this distance is the so-called Korányi-Cygan distance which is left-invariant and homogeneous, i.e. $d_{g}\left(\delta_{\lambda}(\xi), \delta_{\lambda}\left(\xi^{\prime}\right)\right)=\lambda d_{g}\left(\xi, \xi^{\prime}\right)$ for every $\lambda>0, \xi, \xi^{\prime} \in \mathbb{H}^{n}$. We will set $d_{g}(e, \xi)=$ $\|\xi\|_{g}$ for every $\xi \in \mathbb{H}^{n}$. The Korányi-Cygan ball of center $\xi_{0} \in \mathbb{H}^{n}$ and radius $r>0$ is given by $B_{g}\left(\xi_{0}, r\right)=\left\{\xi \in \mathbb{H}^{n}: d_{g}\left(\xi_{0}, \xi\right) \leq r\right\}$.

The horizontal structure relies on the notion of horizontal plane. Given $\xi_{0} \in \mathbb{H}^{n}$, the horizontal plane $H_{\xi_{0}}$ associated to $\xi_{0}=\left(x_{0}, y_{0}, t_{0}\right)$ is the plane in $\mathbb{H}^{n}$ defined by

$$
H_{\xi_{0}}:=\left\{\xi=(x, y, t) \in \mathbb{H}^{n}: t=t_{0}+2\left(y_{0} \cdot x-x_{0} \cdot y\right)\right\}
$$

This is the plane spanned by the horizontal vector fields $\left\{X_{i}, Y_{i}\right\}_{i}$ at the point $\xi_{0}$; note that $\xi^{\prime} \in H_{\xi}$ if and only if $\xi \in H_{\xi^{\prime}}$. A horizontal segment is a convex subset of a horizontal line, which is a line lying on a horizontal plane $H_{\xi}$ and passing though the point $\xi \in \mathbb{H}^{n}$; if $\xi^{\prime} \in H_{\xi}$, with $\xi^{\prime} \neq \xi$, then $H_{\xi} \cap H_{\xi^{\prime}}$ is a horizontal line.

Let $\Omega \subset \mathbb{H}^{n}$ be an open set. The main idea of the analysis in the Heisenberg group is that the regularity properties of functions defined in $\mathbb{H}^{n}$ can be expressed in terms only of the horizontal vector fields (2.13). In particular, the appropriate notion of gradient for a function is the so-called horizontal gradient, which is defined as the $2 n$-vector $\nabla_{H} \varphi(\xi)=\left(X_{1} \varphi(\xi), \ldots, X_{n} \varphi(\xi), Y_{1} \varphi(\xi), \ldots, Y_{n} \varphi(\xi)\right)$ for a function $\varphi \in$ $\Gamma^{1}(\Omega)$. Here, $\Gamma^{k}(\Omega)$ denotes the Folland-Stein space of functions having continuous derivatives up to order $k$ with respect to the vector fields $X_{i}$ and $Y_{i}, i \in\{1, \ldots, n\}$. We say that $\varphi: \Omega \rightarrow \mathbb{R}$ is $H$-differentiable at $\xi$, if there exists a mapping $D_{H} \varphi: \mathbb{H}^{n} \rightarrow \mathbb{R}$ which is $H$-linear, i.e. $D_{H} \varphi(x, y, t)=D_{H} \varphi(x, y, 0)$ for every $(x, y, t) \in \mathbb{H}^{n}$, such that $\varphi\left(\xi \circ \xi^{\prime}\right)=\varphi(\xi)+D_{H} \varphi\left(\xi^{\prime}\right)+o\left(\left\|\xi^{\prime}\right\|_{g}\right)$; the vector associated to $D_{H} \varphi$ with respect to the fixed scalar product is the horizontal gradient $\nabla_{H} \varphi(\xi)$.

For general non-smooth functions $\varphi: \Omega \rightarrow \mathbb{R}$, the horizontal subdifferential $\partial_{H} \varphi\left(\xi_{0}\right)$ of $\varphi$ at $\xi_{0} \in \Omega$ is given by

$$
\partial_{H} \varphi\left(\xi_{0}\right)=\left\{p \in \mathbb{R}^{2 n}: \varphi(\xi) \geq \varphi\left(\xi_{0}\right)+p \cdot\left(\operatorname{Pr}_{1}(\xi)-\operatorname{Pr}_{1}\left(\xi_{0}\right)\right), \forall \xi \in \Omega \cap H_{\xi_{0}}\right\}
$$

where $\operatorname{Pr}_{1}: \mathbb{H}^{n} \rightarrow \mathbb{R}^{2 n}$ is the projection defined by $\operatorname{Pr}_{1}(\xi)=\operatorname{Pr}_{1}(x, y, t)=(x, y)$. It is easy to see that if $\varphi \in \Gamma^{1}(\Omega)$ and $\partial_{H} \varphi(\xi) \neq \emptyset$, then $\partial_{H} \varphi(\xi)=\left\{\nabla_{H} \varphi(\xi)\right\}$. A function $\varphi: \Omega \rightarrow \mathbb{R}$ is called $H$-subdifferentiable on $\Omega$ if $\partial_{H} \varphi(\xi) \neq \emptyset$ for every $\xi \in \Omega$.

A central object of study within this paper is provided by the $H$-convex functions. First of all, we recall that a set $\Omega \subset \mathbb{H}^{n}$ is said to be horizontally convex ( $H$-convex) if, for every $\xi_{1}, \xi_{2} \in \Omega$, with $\xi_{1} \in H_{\xi_{2}}$ and $\lambda \in[0,1]$, we have $\xi_{1} \circ \delta_{\lambda}\left(\xi_{1}^{-1} \circ \xi_{2}\right) \in \Omega$. It is clear that if $\Omega$ is convex (i.e. it is convex in the $\mathbb{R}^{2 n+1}$-sense), then it is also $H$-convex. Given a function $\varphi: \Omega \rightarrow \mathbb{R}$, where $\Omega$ is $H$-convex, there are several equivalent ways to define the concept of $H$-convexity for $\varphi$. The most intuitive one is to require the classical convexity of the function when restricted to any horizontal line within $\Omega$. The same definition can be rephrased by considering the group operation: the function $\varphi: \Omega \rightarrow \mathbb{R}$ is said to be $H$-convex if, for every $\xi_{1}, \xi_{2} \in \Omega$ with $\xi_{1} \in H_{\xi_{2}}$ 
and $\lambda \in[0,1]$, we have that

$$
\varphi\left(\xi_{1} \circ \delta_{\lambda}\left(\xi_{1}^{-1} \circ \xi_{2}\right)\right) \leq(1-\lambda) \varphi\left(\xi_{1}\right)+\lambda \varphi\left(\xi_{2}\right)
$$

If the strict inequality holds in (2.14), for every $\xi_{1} \neq \xi_{2}$ and $\lambda \in(0,1)$, then $\varphi$ is said to be strictly $H$-convex. $H$-convex functions have been extensively studied in the last few years; their characterizations, as well as their regularity properties, like their continuity, for instance, will come into play through the paper, and we refer to $[5,9,15,23]$. Let us recall, in particular, that $\varphi: \mathbb{H}^{n} \rightarrow \mathbb{R}$ is $H$-convex if and only if $\varphi$ is $H$-subdifferentiable.

\section{$3 \mathrm{H}$-Convex Functions with Round $\boldsymbol{H}$-Sections and with Controlled H-Slope}

As already seen in the Introduction, a horizontal notion of section was given in [13] for functions defined on a general Carnot group $\mathbf{G}$. We will consider the particular case $\mathbf{G}=\mathbb{H}^{n}$.

Let $\varphi: \mathbb{H}^{n} \rightarrow \mathbb{R}$ be an $H$-convex function, and let us fix $\xi_{0} \in \mathbb{H}^{n}, p_{0} \in \partial_{H} \varphi\left(\xi_{0}\right)$, and $s>0$. The $H$-section of $\varphi$ at $\xi_{0}, p_{0}$, with height $s$, is the set

$$
S_{\varphi}^{H}\left(\xi_{0}, p_{0}, s\right)=\left\{\xi \in H_{\xi_{0}}: \varphi(\xi)-\varphi\left(\xi_{0}\right)-p_{0} \cdot\left(\operatorname{Pr}_{1}(\xi)-\operatorname{Pr}_{1}\left(\xi_{0}\right)\right)<s\right\} .
$$

If $\varphi$ is $H$-differentiable, then $\partial_{H} \varphi\left(\xi_{0}\right)=\left\{\nabla_{H} \varphi\left(\xi_{0}\right)\right\}$, and we simply write $S_{\varphi}^{H}\left(\xi_{0}, s\right)$ for the corresponding $H$-section. For every fixed $\left(\xi_{0}, p_{0}, s\right)$, the set $S_{\varphi}^{H}\left(\xi_{0}, p_{0}, s\right)$ is $H$-convex, and is contained in a horizontal plane; this dimensional gap between $H$-sections and open sets in $\mathbb{H}^{n}$ is a crucial difference with respect to the Euclidean case.

In this section we essentially introduce the notions of round $H$-sections (see Definition 3.1) and controlled $H$-slope (see Definition 3.2), proving their equivalence (see Theorem 3.1). Let us emphasize that these two properties for an $H$-convex function are horizontal properties, i.e. they give information on the behaviour of the function only when restricted to the horizontal planes, exactly as the notion of $H$-section, $H$-convexity and $H$-subdifferential.

In the following of the paper, for every function $\varphi: \mathbb{H}^{n} \rightarrow \mathbb{R}$, and for every $\xi_{0} \in \mathbb{H}^{n}$, $p_{0} \in \partial_{H} \varphi\left(\xi_{0}\right)$ and $v_{0} \in V_{1} \backslash\{0\}$, we will consider the functions $\varphi_{\xi_{0}, p_{0}}: \mathbb{H}^{n} \rightarrow \mathbb{R}$ and $\widehat{\varphi} \xi_{0}, v_{0}: \mathbb{R} \rightarrow \mathbb{R}$ defined by

$$
\begin{aligned}
& \varphi_{\xi_{0}, p_{0}}(\xi):=\varphi(\xi)-\varphi\left(\xi_{0}\right)-p_{0} \cdot\left(\operatorname{Pr}_{1}(\xi)-\operatorname{Pr}_{1}\left(\xi_{0}\right)\right), \quad \forall \xi \in \mathbb{H}^{n}, \\
& \widehat{\varphi}_{\xi_{0}, v_{0}}(\alpha):=\varphi\left(\xi_{0} \circ \exp \left(\alpha v_{0}\right)\right), \quad \forall \alpha \in \mathbb{R} .
\end{aligned}
$$

If $\varphi$ is $H$-differentiable, then we will set $\varphi_{\xi_{0}}, \nabla_{H} \varphi\left(\xi_{0}\right)=\varphi_{\xi_{0}}$. The following result holds:

Proposition 3.1 Let $\varphi: \mathbb{H}^{n} \rightarrow \mathbb{R}$ be a strictly $H$-convex function. Then, all its $H$ sections are bounded sets. 
Proof For every $\xi_{0} \in \mathbb{H}^{n}$ and $v \in V_{1} \backslash\{0\}$ let us consider the function $\widehat{\varphi}_{\xi_{0}, v}$ as in (3.3). By contradiction, let us suppose that there exists a sequence $\left\{\left(v_{n}, \alpha_{n}\right)\right\}_{n}$, with $v_{n} \in V_{1},\left\|v_{n}\right\|=1, \alpha_{n} \rightarrow+\infty$, such that $\xi_{0} \circ \exp \left(\alpha_{n} v_{n}\right) \in S_{\varphi}^{H}\left(\xi_{0}, p_{0}, s\right)$. Clearly, there exists a subsequence such that $v_{n} \rightarrow v_{0} \in V_{1}$.

Let us denote by $\alpha_{0}=\sup \left\{\alpha \geq 0: \xi_{0} \circ \exp \left(\alpha v_{0}\right) \in \overline{S_{\varphi}^{H}\left(\xi_{0}, p_{0}, s\right)}\right\}$. If $\alpha_{0}=$ $+\infty$, then the section $S_{\widehat{\varphi}_{\xi_{0}, v}}(0, s)$ of the function $\widehat{\varphi}_{\xi_{0}, v}$ is unbounded; this is impossible, since $\widehat{\varphi}_{\xi_{0}, v}$ is strictly convex. Let $s_{0}$ be finite, and let us consider the function $\varphi_{\xi_{0}, p_{0}}$ in (3.2); the set $A=\left\{\xi \in \mathbb{H}^{n}: \varphi_{\xi_{0}, p_{0}}(\xi) \leq s\right\}$ is $H$-convex, since the function $\varphi_{\xi_{0}}, p_{0}$ is $H$-convex. Now, the previous arguments give

$\left\{\xi \in H_{\xi_{0}}: \xi=\xi_{0} \circ \exp \left(\alpha v_{n}\right), 0 \leq \alpha \leq \alpha_{n}\right\} \subset A, \forall n, \quad$ and $\quad \xi^{\prime}=\xi_{0} \circ \exp \left(\alpha_{0} v_{0}\right) \in \partial A$

This contradicts Theorem 1.4 in [3].

The next definition is related to a purely geometric property of the sections, and it will play a crucial role in the following of the paper.

Definition 3.1 We say that an $H$-convex function $\varphi: \mathbb{H}^{n} \rightarrow \mathbb{R}$ has round $H$-sections if there exists a constant $K_{0} \in(0,1)$ with the following property: for every $\xi \in \mathbb{H}^{n}$, $p \in \partial_{H} \varphi(\xi)$ and $s>0$, there exists $R>0$ such that

$$
B_{g}\left(\xi, K_{0} R\right) \cap H_{\xi} \subset S_{\varphi}^{H}(\xi, p, s) \subset B_{g}(\xi, R) \cap H_{\xi} .
$$

In particular, (3.4) implies that every $H$-section of a function with round $H$-sections is a bounded set. Clearly, Definition 3.1 is the $\mathbb{H}^{n}$-version of Definition 2.1; let us stress that it relies upon the subriemannian structure of $\mathbb{H}^{n}$ since, for every point $\xi$, we restrict our attention only to the horizontal plane $H_{\xi}$.

Remark 3.1 Let $\varphi: \mathbb{H}^{n} \rightarrow \mathbb{R}$ be $H$-convex, and consider the convex function $\widehat{\varphi}_{\xi_{0}, v}$ : $\mathbb{R} \rightarrow \mathbb{R}$ defined by (3.3). Then, if the nonempty convex set $\partial_{H} \varphi\left(\xi_{0}\right)$ is not a singleton, there exists $v \in V_{1}$ such that $\partial \widehat{\varphi}_{\xi_{0}, v}(0)$ is not a singleton. Indeed, suppose that $p+\lambda q \in$ $\partial_{H} \varphi\left(\xi_{0}\right)$, for every $\lambda \in[0,1]$, with $q \neq 0$. Then, by taking $v=q$, we have that

$\widehat{\varphi}_{\xi_{0}, q}(\alpha)=\varphi\left(\xi_{0} \circ \exp (\alpha q)\right) \geq \widehat{\varphi}_{\xi_{0}, q}(0)+\alpha\left(p \cdot q+\lambda\|q\|^{2}\right), \quad \forall \lambda \in[0,1], \alpha \in \mathbb{R}$.

Hence $p \cdot q+\lambda\|q\|^{2} \in \partial \widehat{\varphi}_{\xi_{0}, q}(0)$ for every $\lambda \in[0,1]$. This implies that, if $\widehat{\varphi}_{\xi_{0}, v}$ is differentiable at 0 for every $v \in V_{1}$, then $\varphi$ is $H$-differentiable at $\xi_{0}$.

In the previous remark and in the following result, the $H$-convexity plays a fundamental role in order to obtain some regularity properties of the function involved.

Proposition 3.2 If $\varphi: \mathbb{H}^{n} \rightarrow \mathbb{R}$ is an $H$-convex function with round $H$-sections, then it is $H$-differentiable and strictly $H$-convex. Moreover, there exists a constant $C$ such that, for every $\xi_{0} \in \mathbb{H}^{n}$ and $v \in V_{1}$, we have

$$
\varphi_{\xi_{0}}\left(\xi_{0} \circ \exp (2 v)\right) \leq C \varphi_{\xi_{0}}\left(\xi_{0} \circ \exp v\right)
$$

where the constant $C$ depends only on $K_{0}$ in (3.4). 
Proof First of all note that, for every $\xi_{0} \in \mathbb{H}^{n}$ and $v \in V_{1} \backslash\{0\}$, the function $\widehat{\varphi} \xi_{0}, v$ defined in (3.3) is convex, with round sections (with constant $K_{0}$ ). Therefore, Lemma 3.2 in [21] implies that it is differentiable and strictly convex. In particular, $\varphi$ is strictly $H$-convex. Let us first show that $\varphi$ is $H$-differentiable at $\xi_{0} \in \mathbb{H}^{n}$. Since $\varphi$ is $H$-convex, this is equivalent to prove that the nonempty convex set $\partial_{H} \varphi\left(\xi_{0}\right)$ is a singleton (see Theorem 4.4, Prop. 5.1 in [9], Theorem 1.4 in [23]). Suppose, by contradiction, that $\partial_{H} \varphi\left(\xi_{0}\right)$ is not a singleton; then, by Remark 3.1, there exists $v \in V_{1}$ such that $\partial \widehat{\varphi}_{\xi_{0}, v}(0)$ is not a singleton. This contradicts the fact that $\widehat{\varphi}_{\xi_{0}, v}(0)$ is differentiable.

Finally, taking into account that the function $\widehat{\varphi}_{\xi_{0}, v}$ is convex, differentiable and with round sections with constant $K_{0}$, for every $\xi_{0} \in \mathbb{H}^{n}$ and $v \in V_{1}$, again, by Lemma 3.2 in [21], one has that there exists a constant $C$ depending only on $K_{0}$ such that

$$
\varphi_{\xi_{0}}\left(\xi_{0} \circ 2 v\right) \leq C \varphi_{\xi_{0}}\left(\xi_{0} \circ \exp v\right)
$$

In the sequel, given an $H$-differentiable function $\varphi: \mathbb{H}^{n} \rightarrow \mathbb{R}$, we will deal with the functions $m_{\varphi}^{H}, M_{\varphi}^{H}: \mathbb{H}^{n} \times \mathbb{R}^{+} \rightarrow \mathbb{R}^{+}$that will take the place in $\mathbb{H}^{n}$ of the functions $m_{u}$ and $M_{u}$ in $\mathbb{R}^{n}$. They are defined as follows:

$$
\begin{aligned}
& m_{\varphi}^{H}(\xi, r):=\min _{\left\{\xi^{\prime} \in H_{\xi}: d_{g}\left(\xi, \xi^{\prime}\right)=r\right\}}\left(\varphi\left(\xi^{\prime}\right)-\varphi(\xi)-\nabla_{H} \varphi(\xi) \cdot\left(\operatorname{Pr}_{1}\left(\xi^{\prime}\right)-\operatorname{Pr}_{1}(\xi)\right)\right) \\
& M_{\varphi}^{H}(\xi, r):=\max _{\left\{\xi^{\prime} \in H_{\xi}: d_{g}\left(\xi, \xi^{\prime}\right)=r\right\}}\left(\varphi\left(\xi^{\prime}\right)-\varphi(\xi)-\nabla_{H} \varphi(\xi) \cdot\left(\operatorname{Pr}_{1}\left(\xi^{\prime}\right)-\operatorname{Pr}_{1}(\xi)\right)\right),
\end{aligned}
$$

for every $\xi \in \mathbb{H}^{n}, r>0$.

A simple exercise shows that, if $\varphi: \mathbb{H}^{n} \rightarrow \mathbb{R}$ is an $H$-differentiable and strictly $H$-convex function, then for every $\xi \in \mathbb{H}^{n}$, and $r>0$,

$$
S_{\varphi}^{H}\left(\xi, m_{\varphi}^{H}(\xi, r)\right) \subset B_{g}(\xi, r) \cap H_{\xi} \subset S_{\varphi}^{H}\left(\xi, M_{\varphi}^{H}(\xi, r)\right) .
$$

The next definition, inherited from the corresponding one in $\mathbb{R}^{n}$ (see (2.12)), pertains to the mutual behaviour of $m_{\varphi}^{H}$ and $M_{\varphi}^{H}$, always from a horizontal point of view:

Definition 3.2 We say that an $H$-convex function $\varphi: \mathbb{H}^{n} \rightarrow \mathbb{R}$ has controlled $H$-slope if $\varphi$ is $H$-differentiable, and there exists a constant $K_{1}>0$ such that, for every $\xi \in \mathbb{H}^{n}$ and $r>0$,

$$
M_{\varphi}^{H}(\xi, r) \leq K_{1} m_{\varphi}^{H}(\xi, r) .
$$

Like in the Euclidean case (see Theorem 7.1) controlled $H$-slope and round $H$-sections properties are strictly related:

Theorem 3.1 Let $\varphi: \mathbb{H}^{n} \rightarrow \mathbb{R}$ be an $H$-convex function. The following conditions are equivalent:

a. $\varphi$ is an $H$-differentiable function, with bounded $H$-sections and controlled $H$ slope;

b. $\varphi$ has round $H$-sections. 
Moreover, the constants $K_{0}$ and $K_{1}$ in (3.4) and in (3.7) are related, and they depend only on $\varphi$.

Proof Let a. be true. Let $S_{\varphi}^{H}\left(\xi_{0}, s\right)$ be a bounded $H$-section, and let $R=$ $\max \left\{d_{g}\left(\xi, \xi_{0}\right): \xi \in \overline{S_{\varphi}^{H}\left(\xi_{0}, s\right)}\right\}$. Pick a point $\xi^{\prime}$ such that $d_{g}\left(\xi^{\prime}, \xi_{0}\right)=R$; then, $\xi^{\prime} \in \partial S_{\varphi}^{H}\left(\xi_{0}, s\right)$ and $\xi^{\prime}=\xi_{0} \circ \exp v^{\prime}$. From the $H$-convexity of $\varphi_{\xi_{0}}$ on $\mathbb{H}^{n}$, we have that

$$
\varphi_{\xi_{0}}\left(\xi_{0} \circ \exp \left(v^{\prime} / K_{1}\right)\right) \leq\left(1-\frac{1}{K_{1}}\right) \varphi \xi_{0}\left(\xi_{0}\right)+\frac{1}{K_{1}} \varphi \xi_{0}\left(\xi^{\prime}\right)=\frac{s}{K_{1}}
$$

where $K_{1}$ is as in (3.7). Now, for every $\xi \in H_{\xi_{0}}$ such that $d_{g}\left(\xi, \xi_{0}\right)=\frac{R}{K_{1}}$, by (3.7) we have

$$
\varphi_{\xi_{0}}(\xi) \leq M_{\varphi}^{H}\left(\xi_{0}, \frac{R}{K_{1}}\right) \leq K_{1} m_{\varphi}^{H}\left(\xi_{0}, \frac{R}{K_{1}}\right) \leq \varphi_{\xi_{0}}\left(\xi^{\prime}\right) \leq s .
$$

Hence,

$$
B\left(\xi_{0}, \frac{R}{K_{1}}\right) \subset S_{\varphi}^{H}\left(\xi_{0}, s\right) \subset B\left(\xi_{0}, R\right)
$$

Suppose now that condition b. holds true. Proposition 3.2 entails that $\varphi$ is $H$ differentiable. Consider $K_{0}$ as in (3.4), and fix $\xi \in \mathbb{H}^{n}$ and $r>0$ : we have to prove (3.7), where $K_{1}$ is uniform, i.e. it does not depend on $\xi$ and $r$. Set $s=m_{\varphi}^{H}(\xi, r)$ and define

$$
\mathcal{R}=\left\{R^{\prime}>0: B_{g}\left(\xi, K_{0} R^{\prime}\right) \cap H_{\xi} \subset \overline{S_{\varphi}^{H}(\xi, s)} \subset B_{g}\left(\xi, R^{\prime}\right) \cap H_{\xi}\right\} .
$$

Since $\varphi$ has round $H$-sections, $\mathcal{R}$ is not empty. Set $R=\min \mathcal{R}$; trivially, $R=r$, and

$$
\varphi\left(\xi \circ \exp \left(K_{0} v\right)\right)-\varphi(\xi)-\nabla_{H} \varphi(\xi) \cdot\left(K_{0} v\right) \leq s,
$$

for every $v \in V_{1},\|v\|=R$. The two relations above imply that

$$
M_{\varphi}^{H}\left(\xi, K_{0} r\right) \leq m_{\varphi}^{H}(\xi, r) .
$$

Take $\alpha \in \mathbb{N}$ such that $K_{0}>2^{-\alpha}$, and note that relation (3.5) implies

$$
M_{\varphi}^{H}\left(\xi, R_{1}\right) \leq C M_{\varphi}^{H}\left(\xi, R_{1} / 2\right)
$$

for every $R_{1}>0$, where $C$ depends only on $K_{0}$. By iterating this relation, we obtain

$$
\begin{aligned}
M_{\varphi}^{H}(\xi, r) & \leq C M_{\varphi}^{H}\left(\xi, 2^{-1} r\right) \leq C^{2} M_{\varphi}^{H}\left(\xi, 2^{-2} r\right) \\
& \leq \ldots \leq C^{\alpha} M_{\varphi}^{H}\left(\xi, 2^{-\alpha} r\right) \leq C^{\alpha} M_{\varphi}^{H}\left(\xi, K_{0} r\right) .
\end{aligned}
$$


This last inequality, together with (3.8), leads to the assertion, with $K_{1}=C^{-\alpha}$ in (3.7).

In the next result we investigate the properties of the function $m_{\varphi}^{H}$, in order to shed some light on a finer behaviour of the $H$-sections.

Proposition 3.3 Let $\varphi: \mathbb{H}^{n} \rightarrow \mathbb{R}$ be an H-differentiable and strictly H-convex function. For every fixed $\xi \in \mathbb{H}^{n}$, the function $r \mapsto m_{\varphi}^{H}(\xi, r)$ is strictly increasing, continuous, and it goes to $+\infty$, if $r \rightarrow+\infty$. Then, the function $m_{\varphi}^{H}(\xi, \cdot):[0,+\infty) \rightarrow$ $[0,+\infty)$ is one-to-one and onto, and its inverse is defined on $[0,+\infty)$. A similar result holds for the function $M_{\varphi}^{H}$.

Proof For every $\xi \in \mathbb{H}^{n}, r>0$ and $v \in V_{1}$, with $\|v\|=1$, set

$$
\widehat{m}_{\varphi}^{H}(\xi, v, r)=\min \{\varphi(\xi \circ \exp r v), \varphi(\xi \circ \exp (-r) v)\}
$$

The function $\widehat{m}_{\varphi}^{H}$ is continuous, and strictly increasing w.r.t. $r$, since $\varphi$ is strictly $H$-convex; thus,

$$
\widehat{m}_{\varphi}^{H}(\xi, v, r)<\widehat{m}_{\varphi}^{H}\left(\xi, v, r^{\prime}\right), \quad \forall 0 \leq r<r^{\prime}
$$

Hence, by the Berge Maximum Theorem (see, for instance, [2]) $m_{\varphi}^{H}$ is continuous, and

$$
m_{\varphi}^{H}(\xi, r) \leq m_{\varphi}^{H}\left(\xi, r^{\prime}\right), \quad \forall 0 \leq r<r^{\prime}
$$

Let us show that the previous inequality is strict. The set $\left\{v \in V_{1}:\|v\|=1\right\}$ is compact, and $\widehat{m}_{\varphi}^{H}(\xi, \cdot, \cdot)$ is continuous, then there exist $v$ and $v^{\prime}$ such that $\widehat{m}_{\varphi}^{H}(\xi, v, r)=$ $m_{\varphi}^{H}(\xi, r)$ and $\widehat{m}_{\varphi}^{H}\left(\xi, v^{\prime}, r^{\prime}\right)=m_{\varphi}^{H}\left(\xi, r^{\prime}\right)$. This implies that

$$
m_{\varphi}^{H}(\xi, r)=\widehat{m}_{\varphi}^{H}(\xi, v, r) \leq \widehat{m}_{\varphi}^{H}\left(\xi, v^{\prime}, r\right)<\widehat{m}_{\varphi}^{H}\left(\xi, v^{\prime}, r^{\prime}\right)=m_{\varphi}^{H}\left(\xi, r^{\prime}\right) .
$$

Let us show that $m_{\varphi}^{H}(\xi, \cdot)$ is unbounded, for every $\xi$. Suppose, by contradiction, that there exists $L=L(\xi)>0$ such that $m_{\varphi}^{H}(\xi, r) \leq L$ for every $r \geq 0$. From the continuity of the function $v \mapsto \widehat{m}_{\varphi}^{H}(\xi, v, r)$, for every $r$ there exists $v_{r}$, with $\left\|v_{r}\right\|=1$, such that $m_{\varphi}^{H}(\xi, r)=\widehat{m}_{\varphi}^{H}\left(\xi, v_{r}, r\right)$. Let $r_{n} \rightarrow+\infty$; then, there exists $\left\{v_{r_{n_{k}}}\right\}$ such that $v_{r_{n_{k}}} \rightarrow \bar{v}$. We have that

$$
\lim _{k \rightarrow+\infty} \widehat{m}_{\varphi}^{H}\left(\xi, v_{r_{n_{k}}}, r_{n_{k}}\right)=\lim _{k \rightarrow+\infty} \widehat{m}_{\varphi}^{H}\left(\xi, \bar{v}, r_{n_{k}}\right)=+\infty
$$

contradicting the assumption that $m_{\varphi}^{H}(\xi, r)=\widehat{m}_{\varphi}^{H}\left(\xi, v_{r}, r\right) \leq L$ for every $r>0$. 


\section{Engulfing Property for $\boldsymbol{H}$-Sections of $\boldsymbol{H}$-Convex Functions}

This section is devoted to the study of the engulfing property $E(H, K)$ for the $H$ sections of an $H$-convex function. Our notion is different when compared with the one introduced by Capogna and Maldonado, and it generalizes the usual notion in the literature (see for example [19]); however, we will see that these notions are equivalent (see Proposition 4.2). In the second part of the section we prove that a sufficient condition for a function to satisfy the engulfing property $E(H, K)$ is to have the round $H$-sections property, or, equivalently, the controlled $H$-slope (see Theorem 3.1). Finally, we will show, with an example, that the previous mentioned condition is only sufficient.

Let us start with our notion of engulfing for $H$-convex functions defined in $\mathbb{H}^{n}$.

Definition 4.1 Let $\varphi: \mathbb{H}^{n} \rightarrow \mathbb{R}$ be an $H$-convex function. We say that $\varphi$ satisfies the engulfing property $E(H, K)$ (shortly, $\varphi \in E(H, K)$ ) if there exists $K>1$ such that, for any $\xi \in \mathbb{H}^{n}$ and $s>0$, if $\xi^{\prime} \in S_{\varphi}^{H}(\xi, p, s)$ with $p \in \partial_{H} \varphi(\xi)$, then

$$
S_{\varphi}^{H}(\xi, p, s) \cap H_{\xi^{\prime}} \subset S_{\varphi}^{H}\left(\xi^{\prime}, q, K s\right) \cap H_{\xi},
$$

for every $q \in \partial_{H} \varphi\left(\xi^{\prime}\right)$.

As a matter of fact, as mentioned previously, in [13] a slightly different definition of engulfing is investigated in the framework of Carnot groups; if $\mathbf{G}=\mathbb{H}^{n}$, it can be stated as follows:

$$
\left\{\begin{array}{l}
\exists K>1: \text { for every } \xi, \xi^{\prime} \in \mathbb{H}^{n} \text { and } s>0, \\
\text { if } \xi^{\prime} \in S_{\varphi}^{H}(\xi, s), \text { then } \xi \in S_{\varphi}^{H}\left(\xi^{\prime}, K s\right)
\end{array}\right.
$$

(we will refer to $\left(\mathbf{e n g}_{H}\right)_{K}$ in case the constant $K$ plays a role). Trivially, $\varphi \in$ $E(H, K)$ implies that $\varphi$ satisfies $\left(\mathbf{e n g}_{H}\right)_{K}$. The condition $\left(\mathbf{e n g}_{H}\right)$ is essentially onedimensional, as proved in the next

Proposition 4.1 (see [13]). Let $\varphi: \mathbb{H}^{n} \rightarrow \mathbb{R}$ be a strictly $H$-convex and $H$ differentiable function. The function $\varphi$ satisfies $\left(\mathbf{e n g}_{H}\right)_{K}$ if and only iffor every $\xi \in \mathbb{H}^{n}$ and $v \in V_{1}$ the function $\varphi_{\xi, v}: \mathbb{R} \rightarrow \mathbb{R}$ satisfies condition ii. in Theorem 2.1. 2.1:

The following characterization provides an $\mathbb{H}^{n}$-version of the result in Theorem

Proposition 4.2 Let $\varphi: \mathbb{H}^{n} \rightarrow \mathbb{R}$ be a strictly $H$-convex function. The following are equivalent:

i. $\varphi$ satisfies the engulfing property $E(H, K)$, for some $K>1$;

ii. $\varphi$ satisfies condition $\left(\mathbf{e n g}_{H}\right)_{K^{\prime}}$, for some $K^{\prime}>1$; 
iii. there exists a constant $K^{\prime \prime}>1$ such that, for any $\xi \in \mathbb{H}^{n}, \xi^{\prime} \in H_{\xi}$, for any $p \in \partial_{H} \varphi(\xi)$ and $q \in \partial_{H} \varphi\left(\xi^{\prime}\right)$,

$$
\begin{aligned}
& \frac{K^{\prime \prime}+1}{K^{\prime \prime}}\left(\varphi\left(\xi^{\prime}\right)-\varphi(\xi)-p \cdot\left(\operatorname{Pr}_{1}\left(\xi^{\prime}\right)-\operatorname{Pr}_{1}(\xi)\right)\right) \\
& \leq(q-p) \cdot\left(\operatorname{Pr}_{1}\left(\xi^{\prime}\right)-\operatorname{Pr}_{1}(\xi)\right) \\
& \leq\left(K^{\prime \prime}+1\right)\left(\varphi\left(\xi^{\prime}\right)-\varphi(\xi)-p \cdot\left(\operatorname{Pr}_{1}\left(\xi^{\prime}\right)-\operatorname{Pr}_{1}(\xi)\right)\right) .
\end{aligned}
$$

In particular, if any of the conditions above holds, $\varphi$ is H-differentiable.

Proof Trivially, i. implies ii., and one can take $K^{\prime}=K$. Let us show that ii. implies i. Let $\xi^{\prime}=\xi \circ \exp v$ be a point in $S_{\varphi}^{H}(\xi, p, s)$, and consider the convex function $\widehat{\varphi}_{\xi, v}: \mathbb{R} \rightarrow \mathbb{R}$ defined in (3.3). Note that

$$
S_{\varphi}^{H}(\xi, p, s) \cap H_{\xi^{\prime}}=\left\{\xi \circ \exp s v: s \in S_{\widehat{\varphi}_{\xi, v}}(0, p \cdot v, s)\right\},
$$

and the function $\widehat{\varphi} \xi, v$ satisfies condition ii. in Th. 2.1 with constant $K^{\prime}$. From Theorem 1 in [11], $\widehat{\varphi} \xi, v \in C^{1}(\mathbb{R})$. Since this holds for every $\xi, v$, from Remark $3.1 \varphi$ is $H$ differentiable everywhere and $\partial_{H} \varphi(\xi)=\left\{\nabla_{H} \varphi(\xi)\right\}$. Moreover, from Theorem 5.1 in [14], the function $\widehat{\varphi}_{\xi, v}$ satisfies the engulfing condition with constant $2 K^{\prime}\left(K^{\prime}+1\right)$. This is equivalent to say that

$$
\begin{aligned}
& \left\{\alpha \in \mathbb{R}: \widehat{\varphi}_{\xi, v}(\alpha)-\widehat{\varphi}_{\xi, v}(0)-\widehat{\varphi}_{\xi, v}^{\prime}(0) \alpha<s\right\} \\
& \quad \subset\left\{\alpha \in \mathbb{R}: \widehat{\varphi}_{\xi, v}(\alpha)-\widehat{\varphi}_{\xi, v}(1)-\widehat{\varphi}_{\xi, v}^{\prime}(1)(\alpha-1)<2 K^{\prime}\left(K^{\prime}+1\right) s\right\} .
\end{aligned}
$$

From (4.1), we get that

$$
\begin{aligned}
& \left\{\alpha \in \mathbb{R}: \varphi(\xi \circ \exp \alpha v)-\varphi(\xi)-\nabla_{H} \varphi(\xi) \cdot v \alpha<s\right\} \\
& \quad \subset\left\{\alpha \in \mathbb{R}: \varphi(\xi \circ \exp \alpha v)-\varphi(\xi \circ \exp v)-\nabla_{H} \varphi(\xi \circ \exp v) \cdot v(\alpha-1)<2 K^{\prime}\left(K^{\prime}+1\right) s\right\},
\end{aligned}
$$

i.e., $\varphi$ is in $E\left(H, 2 K^{\prime}\left(K^{\prime}+1\right)\right)$.

In order to prove that ii. implies iii., let $\xi^{\prime}=\xi \circ \exp v$ and consider the convex function $\widehat{\varphi}_{\xi, v}$. Note that $p \cdot v \in \partial \widehat{\varphi}_{\xi, v}(0)$ and $q \cdot v \in \partial \widehat{\varphi}_{\xi, v}(1)$. Then, by applying Proposition 2.1 in [11], we have that iii. holds with $K^{\prime \prime}=K^{\prime}$. To conclude, let us show that iii. implies ii. Take $\xi^{\prime}=\xi \circ \exp v \in S_{\varphi}^{H}(\xi, p, s)$, where $p \in \partial_{H} \varphi(\xi)$, and let $q \in \partial_{H} \varphi\left(\xi^{\prime}\right)$. Then,

$$
\varphi(\xi)-\varphi(\xi \circ \exp v)-q \cdot(-v) \leq \frac{K^{\prime \prime}}{K^{\prime \prime}+1}(q-p) \cdot v
$$

The second inequality in iii. gives

$$
(p-q) \cdot(-v) \leq\left(K^{\prime \prime}+1\right)(\varphi(\xi \circ \exp v)-\varphi(\xi)-p \cdot v) \leq\left(K^{\prime \prime}+1\right) s
$$


Then,

$$
\varphi(\xi)-\varphi(x \circ \exp v)-q \cdot(-v) \leq K^{\prime \prime} s,
$$

thus, $\xi \in S_{\varphi}^{H}\left(\xi \circ \exp v, q, K^{\prime \prime} s\right)$, i.e., condition $\left(\mathbf{e n g}_{H}\right)_{K^{\prime \prime}}$ is fulfilled.

Let us recall that a set-valued map $T: \mathbb{H}^{n} \rightarrow \mathcal{P}\left(V_{1}\right)$ is said to be $H$-monotone if, for all $\xi \in \mathbb{H}^{n}, \xi^{\prime} \in H_{\xi}, p \in T(\xi), q \in T\left(\xi^{\prime}\right)$, then

$$
(q-p) \cdot\left(\operatorname{Pr}_{1}\left(\xi^{\prime}\right)-\operatorname{Pr}_{1}(\xi)\right) \geq 0
$$

(here $V_{1} \cong \mathbb{R}^{2 n}$ ) In particular, if $\varphi$ is an $H$-convex function, then the $H$-subdifferential map $\partial_{H} \varphi$ is an $H$-monotone set-valued map (see [10]). The property iii. above requires, in fact, a stronger control on the $H$-monotonicity, both from below and from above.

Let us now state the following crucial result, that provides a sufficient condition for $E(H, K)$ via the round $H$-sections property; the relationship between round $H$ sections, or, equivalently, controlled $H$-slope, and the engulfing property corresponds to the similar one in $\mathbb{R}^{n}$, for $n \geq 2$ :

Theorem 4.1 If $\varphi: \mathbb{H}^{n} \rightarrow \mathbb{R}$ is an $H$-convex function with round $H$-sections, then $\varphi$ satisfies the engulfing property $E(H, K)$, where $K$ depends only on $K_{0}$ in (3.4).

Proof Since $\varphi$ has round $H$-sections, Proposition 3.2 implies that $\varphi$ is strictly $H$ convex and $H$-differentiable. Let $\xi^{\prime} \in S_{\varphi}^{H}(\xi, s)$ be such that $\xi^{\prime}=\xi \circ \exp \left(r^{\prime} v\right)$ for some $v$ in $V_{1}$, with $\|v\|=1$ and $r^{\prime}>0$; we will prove that $\xi \in S_{\varphi}^{H}\left(\xi^{\prime}, K s\right)$ where $K$ depends only on $K_{0}$ in (3.4).

Let $R$ be such that

$$
B_{g}\left(\xi, K_{0} R\right) \cap H_{\xi} \subset S_{\varphi}^{H}(\xi, s) \subset B_{g}(\xi, R) \cap H_{\xi}
$$

Since $S_{\varphi}^{H}(\xi, s)$ is bounded, let us consider

$$
r^{\partial}=\max \left\{r \geq 0: \xi \circ \exp (r v) \in \overline{S_{\varphi}^{H}(\xi, s)}\right\}, \quad \xi^{\partial}=\xi \circ \exp \left(r^{\partial} v\right) \in \partial S_{\varphi}^{H}(\xi, s) .
$$

Hence,

$$
K_{0} R \leq r^{\partial} \leq R
$$

and $0<r^{\prime} \leq r^{\partial}$. From the $H$-monotonicity of the map $\xi \mapsto \partial_{H} \varphi(\xi)$ we have that

$$
0 \leq\left(\nabla_{H} \varphi\left(\xi^{\prime}\right)-\nabla_{H} \varphi(\xi)\right) \cdot v \leq\left(\nabla_{H} \varphi\left(\xi^{\partial}\right)-\nabla_{H} \varphi(\xi)\right) \cdot v
$$

Let us introduce the function $\Phi: \mathbb{R} \rightarrow \mathbb{R}$ defined by

$$
\Phi(\alpha)=\widehat{\varphi} \xi, v(\alpha), \quad \forall \alpha \in \mathbb{R}
$$


this function is strictly convex, with $\Phi(0)=\Phi^{\prime}(0)=0$. Let us consider the function $\Pi: \mathbb{R} \rightarrow \mathbb{R}$ defined by

$$
\Pi(\alpha)=\Phi\left(r^{\partial}\right)+\Phi^{\prime}\left(r^{\partial}\right)\left(\alpha-r^{\partial}\right), \quad \forall \alpha \in \mathbb{R} ;
$$

clearly, it represents the tangent to the graph of $\Phi$ at $\left(r^{\partial}, \Phi\left(r^{\partial}\right)\right)$ with $\Phi\left(r^{\partial}\right)>0$ and $\Phi^{\prime}\left(r^{\partial}\right)>0$; hence we have

$$
\begin{aligned}
\Pi(\alpha)= & \varphi\left(\xi \circ \exp \left(r^{\partial} v\right)\right)-\varphi(\xi)-\nabla_{H} \varphi(\xi) \cdot v r^{\partial}+ \\
& +\left(\nabla_{H} \varphi\left(\xi \circ \exp \left(r^{\partial} v\right)\right)-\nabla_{H} \varphi(\xi)\right) \cdot v\left(\alpha-r^{\partial}\right) \\
= & \varphi(\xi \circ \exp (\alpha v))-\varphi(\xi)-\nabla_{H} \varphi(\xi) \cdot v \alpha+ \\
& -\left(\varphi(\xi \circ \exp (\alpha v))-\varphi\left(\xi \circ \exp \left(r^{\partial} v\right)\right)-\nabla_{H} \varphi\left(\xi \circ \exp \left(r^{\partial} v\right)\right) \cdot v\left(\alpha-r^{\partial}\right)\right)
\end{aligned}
$$

Since $\varphi$ is $H$-convex, the previous equalities and (3.2) give

$$
\begin{aligned}
& \Pi(\alpha) \geq\left(\nabla_{H} \varphi\left(\xi^{\partial}\right)-\nabla_{H} \varphi(\xi)\right) \cdot v\left(\alpha-r^{\partial}\right), \quad \forall \alpha \\
& \Pi(\alpha) \leq \varphi_{\xi}(\xi \circ \exp (\alpha v)), \quad \forall \alpha
\end{aligned}
$$

From (4.4) and (4.5) we get

$$
\left(\nabla_{H} \varphi\left(\xi^{\prime}\right)-\nabla_{H} \varphi(\xi)\right) \cdot v \leq\left(\nabla_{H} \varphi\left(\xi^{\partial}\right)-\nabla_{H} \varphi(\xi)\right) \cdot v \leq \frac{\Pi\left(2 r^{\partial}\right)}{r^{\partial}}
$$

The inequality above, together with (4.3) and (4.6), give

$$
\left(\nabla_{H} \varphi\left(\xi^{\prime}\right)-\nabla_{H} \varphi(\xi)\right) \cdot v \leq \frac{\varphi_{\xi}\left(\xi \circ \exp \left(2 r^{\partial} v\right)\right)}{K_{0} R} .
$$

The $H$-convexity of $\varphi$ and (4.3) imply that $\varphi_{\xi}\left(\xi \circ \exp \left(2 r^{\partial} v\right)\right) \leq \varphi_{\xi}(\xi \circ \exp (2 R v))$; hence we obtain

$$
\left(\nabla_{H} \varphi\left(\xi^{\prime}\right)-\nabla_{H} \varphi(\xi)\right) \cdot v \leq \frac{\varphi_{\xi}(\xi \circ \exp (2 R v))}{K_{0} R} .
$$

Let us consider $\alpha \in \mathbb{N}$ such that $K_{0}>2^{-\alpha}$. By iterating relation (3.5), we obtain

$$
\begin{aligned}
\varphi_{\xi}(\xi \circ \exp (2 R v)) & \leq C \varphi_{\xi}(\xi \circ \exp (R v)) \\
& \leq C^{1+\alpha} \varphi_{\xi}\left(\xi \circ \exp \left(R 2^{-\alpha} v\right)\right) \leq C^{1+\alpha} \varphi_{\xi}\left(\xi \circ \exp \left(K_{0} R v\right)\right)
\end{aligned}
$$

where $C$ depends only on $K_{0}$. The previous inequality, and relations (4.2) and (4.7), give

$$
\left(\nabla_{H} \varphi\left(\xi^{\prime}\right)-\nabla_{H} \varphi(\xi)\right) \cdot v \leq C^{1+\alpha} \frac{s}{K_{0} R} .
$$


At this point, since $S_{\varphi}^{H}(\xi, s)$ is open, there exists $\tilde{\xi}=\xi \circ \exp (\tilde{r} v) \in S_{\varphi}^{H}(\xi, s)$ with $\tilde{r}<0$. Taking into account that $\xi^{\prime} \in S_{\varphi}^{H}(\xi, s)$ and $\tilde{\xi} \in S_{\varphi}^{H}(\xi, s)$, and using (4.8) and (4.2), we obtain

$$
\begin{aligned}
& \varphi(\tilde{\xi})-\varphi\left(\xi^{\prime}\right)-\nabla_{H} \varphi\left(\xi^{\prime}\right) \cdot\left(\operatorname{Pr}_{1}(\tilde{\xi})-\operatorname{Pr}_{1}\left(\xi^{\prime}\right)\right)= \\
& =\varphi(\tilde{\xi})-\varphi(\xi)-\nabla_{H} \varphi(\xi) \cdot\left(\operatorname{Pr}_{1}(\tilde{\xi})-\operatorname{Pr}_{1}(\xi)\right)+ \\
& \quad-\left(\varphi\left(\xi^{\prime}\right)-\varphi(\xi)-\nabla_{H} \varphi(\xi) \cdot\left(\operatorname{Pr}_{1}\left(\xi^{\prime}\right)-\operatorname{Pr}_{1}(\xi)\right)\right)+ \\
& \quad-\left(\nabla_{H} \varphi\left(\xi^{\prime}\right)-\nabla_{H} \varphi(\xi)\right) \cdot\left(\operatorname{Pr}_{1}(\tilde{\xi})-\operatorname{Pr}_{1}\left(\xi^{\prime}\right)\right) \\
& \quad<s+\left(\nabla_{H} \varphi\left(\xi^{\prime}\right)-\nabla_{H} \varphi(\xi)\right) \cdot v\left(r^{\prime}-\tilde{r}\right) \\
& \quad \leq s\left(1+\frac{2 C^{1+\alpha}}{K_{0}}\right) .
\end{aligned}
$$

This implies that $\tilde{\xi} \in S_{\varphi}^{H}\left(\xi^{\prime}, K s\right)$, with $K=1+\frac{2 C^{1+\alpha}}{K_{0}}$ : since $\xi$ belongs to the horizontal segment which joins $\tilde{\xi}$ and $\xi^{\prime}$, and since $S_{\varphi}^{H}\left(\xi^{\prime}, K s\right)$ is $H$-convex, then $\xi \in S_{\varphi}^{H}\left(\xi^{\prime}, K s\right)$. By Proposition 4.2 the assertion is proved.

The following example is crucial in order to shed some light on the relationship between round sections and engulfing; indeed, it shows that the converse of the previous theorem fails. The idea is taken from an example due to Wang (see [24]) and set in $\mathbb{R}^{2}$; we adapt his idea to the case of the first Heisenberg group $\mathbb{H}$.

Example 4.1 Consider the following differentiable and strictly convex function $u$ : $\mathbb{R}^{2} \rightarrow \mathbb{R}$

$$
u(x, y)= \begin{cases}x^{4}+\frac{3 y^{2}}{2 x^{2}} & |y| \leq|x|^{3} \\ \frac{1}{2} x^{2}|y|^{2 / 3}+2|y|^{4 / 3} & |y|>|x|^{3} .\end{cases}
$$

The Monge-Ampère measure $\mu_{u}$ (we recall that $\mu_{u}$ is defined by $\mu_{u}(E)=|\partial u(E)|$ for every Borel set $E \subset \mathbb{R}^{2}$ ) is absolutely continuous with respect to the Lebesgue measure $|\cdot|$, and it verifies the condition $\mu_{\infty}$, i.e. for any $\delta_{1} \in(0,1)$ there exists $\delta_{2} \in(0,1)$ such that: for every section $S_{u}(z, s)$, with $z \in \mathbb{R}^{2}$, and for every Borel set $B \subset S_{u}(z, s)$,

$$
\frac{|B|}{\left|S_{u}(z, s)\right|}<\delta_{2} \Rightarrow \frac{\mu_{u}(B)}{\mu_{u}\left(S_{u}(z, s)\right)}<\delta_{1}
$$

(see Definition 3.7 in [21]). This condition $\mu_{\infty}$ is stronger than the (DC)-doubling property (see, for example, relation (3.1.1) in [19]), i.e., there exist constants $\alpha \in(0,1)$ and $C>1$ such that

$$
\mu_{u}\left(S_{u}(z, s)\right) \leq C \mu_{u}\left(\alpha S_{u}(z, s)\right),
$$

for every $z, s>0$ (here $\alpha S_{u}(z, s)$ denotes the open convex set obtained by $\alpha$ contraction of $S_{u}(z, \tau)$ with respect to its center of mass). In [20] and [17] it was 
shown that the (DC)-doubling property of the measure $\mu_{u}$ is equivalent to the engulfing property of the function $u$. Therefore, $u$ satisfies the engulfing property.

Since the second derivative of $u$ w.r.t. $x_{2}$ is unbounded near the origin, so is $\left\|D^{2} u\right\|$; thus, $u$ is not quasiuniformly convex (see Theorem 7.1-i. and [21] for further details). However, a simpler argument can be advanced to prove that $u$ is not quasiuniformly convex, that is one can show that $u$ has not controlled slope in (2.12): in order to do that, we only remark that, taking into account that $u(0,0)=0$ and $\nabla u(0,0)=(0,0)$, we have, for large $r$,

$$
\begin{aligned}
& m_{u}((0,0), r)=\min _{\left\{z \in \mathbb{R}^{2}:\|z\|=r\right\}} u(z) \leq \varphi(0, r)=2 r^{4 / 3} \\
& M_{u}((0,0), r)=\max _{\left\{z \in \mathbb{R}^{2}:\|z\|=r\right\}} u(z) \geq \varphi(r, 0)=r^{4} .
\end{aligned}
$$

Now let us consider the function $\varphi: \mathbb{H} \rightarrow \mathbb{R}$ defined by $\varphi(x, y, t)=u(x, y)$, for all $(x, y, t) \in \mathbb{H}$. This function $\varphi$ is $\mathbb{R}^{3}$-convex, and hence $H$-convex. Since

$$
m_{u}((0,0), r)=m_{\varphi}^{H}((0,0,0), r), \quad M_{u}((0,0), r)=M_{\varphi}^{H}((0,0,0), r) .
$$

$\varphi$ has not controlled $H$-slope, and hence has not round $H$-sections. However, since

$$
(x, y) \in S_{u}\left(\left(x_{0}, y_{0}\right), s\right) \quad \Longleftrightarrow \quad(x, y, t) \in S_{\varphi}^{H}\left(\left(x_{0}, y_{0}, t_{0}\right), s\right),
$$

it is easy to see that $\varphi$ enjoys the engulfing property.

\section{$5 \mathbb{H}^{n}$-Sections of $\boldsymbol{H}$-Convex Functions and Their Engulfing Properties}

In this section we will present our new definition of section in $\mathbb{H}^{n}$. First of all, we will prove that these $\mathbb{H}^{n}$-sections are full-dimensional, i.e., they contain a Korányi-Cygan ball. This allows to construct a topology in $\mathbb{H}^{n}$, as we will see in the next Sect. 6. In the second part, we introduce the condition of engulfing $E\left(\mathbb{H}^{n}, K\right)$ for these new $\mathbb{H}^{n}$ sections. It will not be a surprise that $\varphi \in E\left(\mathbb{H}^{n}, K\right)$ implies that $\varphi \in E(H, K)$, while the converse implication is very hard and mysterious (at least to us). In order to shed some light on this, let us focus our attention on the functions having round $H$-sections, or, equivalently, controlled $H$-slope. As we will see, some technical estimates allow us to prove the first part of our main result in Theorem 1.1.

Let us start with our new notion of $\mathbb{H}^{n}$-section:

Definition 5.1 Let $\varphi: \mathbb{H}^{n} \rightarrow \mathbb{R}$ be an $H$-convex function and let us fix $\xi_{0} \in \mathbb{H}^{n}$. For a given $s>0$, an $\mathbb{H}^{n}$-section of $\varphi$ at height $s$, with $p_{0} \in \partial_{H} \varphi\left(\xi_{0}\right)$, is the set

$$
\begin{aligned}
& \mathbb{S}_{\varphi}^{\mathbb{H}^{n}}\left(\xi_{0}, p_{0}, s\right)= \\
& \xi_{1} \in S_{\varphi}^{H}\left(\xi_{0}, p_{0}, s\right), p_{1} \in \partial_{H} \varphi\left(\xi_{1}\right), \\
& \xi_{2} \in S_{\varphi}^{H}\left(\xi_{1}, p_{1}, s\right), p_{2} \in \partial_{H} \varphi\left(\xi_{2}\right)
\end{aligned}
$$


In case $\varphi$ is $H$-differentiable at $\xi_{0}$, we will denote the $\mathbb{H}^{n}$-section at $\xi_{0}$ with height $s$ by $\mathbb{S}_{\varphi}^{\mathbb{H}^{n}}\left(\xi_{0}, s\right)$, for short.

Let us spend a few words on the definition above. Lemma 1.40 in the fundamental book by Folland and Stein [16] guarantees that, in every stratified group $(\mathbf{G}, \circ)$ with homogeneous norm $\|\cdot\|_{\mathbf{G}}$, there exists a constant $C>0$ and an integer $k \in \mathbb{N}$ such that any $\xi \in \mathbf{G}$ can be expressed as $\xi=\xi_{1} \circ \xi_{2} \circ \ldots \circ \xi_{k}$, with $\xi_{i} \in \exp \left(V_{1}\right)$ and $\left\|\xi_{i}\right\|_{\mathbf{G}} \leq C\|\xi\|_{\mathbf{G}}$, for every $i$. If $\mathbf{G}=\mathbb{H}^{n}$, the mentioned $k$ is exactly 3 , for every $n \geq 1$. In other words, every point $\xi \in \mathbb{H}^{n}$ can be reached from the origin $e$ following a path of three consecutive horizontal segments. The idea behind Definition 5.1 takes inspiration from this result, in view of providing a family of sets with nonempty interior. Let us define, for every $\xi \in \mathbb{H}^{n}$ and $r>0$,

$$
\widetilde{B}(\xi, r)=\left\{\xi^{\prime} \in \mathbb{H}^{n}: \xi^{\prime}=\xi \circ \exp \left(v_{1}\right) \circ \exp \left(v_{2}\right) \circ \exp \left(v_{3}\right) ; v_{i} \in V_{1},\left\|v_{i}\right\| \leq r\right\}
$$

Clearly, $\delta_{\lambda}(\widetilde{B}(e, r))=\widetilde{B}(e, \lambda r)$, and the associated distance $\widetilde{d}$ in $\mathbb{H}^{n}$ is left-invariant and homogeneous; hence, it is bi-Lipschitz equivalent to $d_{g}$ and to any other leftinvariant and homogeneous distance in $\mathbb{H}^{n}$. Moreover, due to the Folland-Stein Lemma, we have that, for every $\xi \in \mathbb{H}^{n}$ and $r>0$,

$$
\widetilde{B}(\xi, r) \subset B_{g}(\xi, 3 r) \subset \widetilde{B}(\xi, 3 C r)
$$

where $C$ is the constant in the mentioned lemma.

Let us prove the first fundamental property of the $\mathbb{H}^{n}$-sections, namely $\mathbb{S}_{\varphi}^{\mathbb{H}^{n}}\left(\xi_{0}, p_{0}, s\right)$ is full-dimensional.

Proposition 5.1 Let $\varphi: \mathbb{H}^{n} \rightarrow \mathbb{R}$ be an $H$-convex function. Then, for every $\xi_{0} \in$ $\mathbb{H}^{n}, p_{0} \in \partial_{H} \varphi\left(\xi_{0}\right)$ and $s>0$, there exists $r>0$ such that

$$
B_{g}\left(\xi_{0}, r\right) \subset \mathbb{S}_{\varphi}^{\mathbb{H}^{n}}\left(\xi_{0}, p_{0}, s\right)
$$

Proof Without loss of generality, we set $\xi_{0}=e$. Since the $H$-subdifferential map $\partial_{H} \varphi$ brings compact sets into compact sets (see, for instance, Proposition 2.1 in [4]), there exists a positive constant $R$ such that

$$
\partial_{H} \varphi(\widetilde{B}(e, 1)) \subset B^{\mathbb{R}^{2 n}}(0, R) .
$$

Moreover, since $\varphi$ is locally Lipschitz (see Theorem 1.2 in [5]), there exists a positive constant $L$ such that

$$
\left|\varphi(\xi)-\varphi\left(\xi^{\prime}\right)\right| \leq L d_{g}\left(\xi, \xi^{\prime}\right), \quad \forall \xi, \xi^{\prime} \in \widetilde{B}(e, 1)
$$

Set

$$
r=\min \left\{\frac{s}{(L+R) C}, \frac{1}{C}\right\},
$$


where $C$ is the constant in the Folland-Stein Lemma. We will show that $B_{g}(e, r) \subset$ $\mathbb{S}_{\varphi}^{\mathbb{H}^{n}}\left(e, p_{0}, s\right)$.

Take any $\xi \in B_{g}(e, r)$; then, $\xi=\exp \left(v_{1}\right) \circ \exp \left(v_{2}\right) \circ \exp \left(v_{3}\right)$ for suitable $\left\{v_{i}\right\}_{i=1}^{3} \subset$ $V_{1}$ such that $\left\|v_{i}\right\| \leq C\|\xi\|_{g}$, for $i=1,2,3$. Set

$$
\xi_{1}:=\exp \left(v_{1}\right), \quad \xi_{2}:=\xi_{1} \circ \exp \left(v_{2}\right), \quad \xi=\xi_{3}:=\xi_{2} \circ \exp \left(v_{3}\right)
$$

Note that $\xi_{i} \in \widetilde{B}(e, r)$ for $i=1,2,3$. Then, for every $p_{i} \in \partial_{H} \varphi\left(\xi_{i}\right)(i=1,2)$, by (5.4) and (5.5) we have

$$
\varphi(\xi)-\varphi\left(\xi_{2}\right)-p_{2} \cdot v_{3} \leq(L+R)\left\|v_{3}\right\|<s
$$

and

$$
\varphi\left(\xi_{2}\right)-\varphi\left(\xi_{1}\right)-p_{1} \cdot v_{2} \leq(L+R)\left\|v_{2}\right\|<s .
$$

Since $\varphi\left(\xi_{1}\right)-\varphi(e)-p_{0} \cdot v_{1} \leq(L+R)\left\|v_{1}\right\|<s$, from (5.6) and (5.7) we get the claim.

Starting from these $\mathbb{H}^{n}$-sections, we introduce the following engulfing property:

Definition 5.2 Let $\varphi: \mathbb{H}^{n} \rightarrow \mathbb{R}$ be an $H$-convex function. We say that $\varphi$ satisfies the engulfing property $E\left(\mathbb{H}^{n}, K\right)$ if there exists $K>1$ such that, for any $\xi \in \mathbb{H}^{n}, p \in$ $\partial_{H} \varphi(\xi)$ and $s>0$, if $\xi^{\prime} \in \mathbb{S}_{\varphi}^{\mathbb{H}^{n}}(\xi, p, s)$, then

$$
\mathbb{S}_{\varphi}^{\mathbb{H}^{n}}(\xi, p, s) \subset \mathbb{S}_{\varphi}^{\mathbb{H}^{n}}\left(\xi^{\prime}, q, K s\right)
$$

for every $q \in \partial_{H} \varphi\left(\xi^{\prime}\right)$.

The engulfing property $E(H, K)$ is related to this engulfing property $E\left(\mathbb{H}^{n}, K\right)$ as well as condition $\left(\mathbf{e n g}_{H}\right)$ is related to the following condition:

$$
\left\{\begin{array}{l}
\exists K>1: \text { for every } \xi \in \mathbb{H}^{n}, p \in \partial_{H} \varphi(\xi) \text { and } s>0 \\
\text { if } \xi^{\prime} \in \mathbb{S}_{\varphi}^{\mathbb{H}^{n}}(\xi, p, s) \text {, then } \xi \in \mathbb{S}_{\varphi}^{\mathbb{H}^{n}}\left(\xi^{\prime}, q, K^{\prime} s\right) \text { for every } q \in \partial_{H} \varphi\left(\xi^{\prime}\right)
\end{array}\right.
$$

$\left(\mathbf{e n g}_{\mathbb{H}^{n}}\right)$

We will refer to $\left(\mathbf{e n g}_{\mathbb{H}^{n}}\right)_{K}$ in case we need to specify the constant $K$ in the previous condition.

It is clear that

Remark 5.1 If $\varphi$ satisfies the engulfing property $E\left(\mathbb{H}^{n}, K\right)$, then condition $\left(\mathbf{e n g}_{\mathbb{H}^{n}}\right)_{K}$ holds.

The converse of the previous remark is a delicate question: the aim of this section is, essentially, to prove that, under further conditions on $\varphi$, the converse of Remark 5.1 holds.

The relationship between conditions $\left(\mathbf{e n g}_{H}\right)$ and $\left(\mathbf{e n g}_{\mathbb{H}^{n}}\right)$ is the following: 
Proposition 5.2 Let $\varphi: \mathbb{H}^{n} \rightarrow \mathbb{R}$ be an $H$-convex function. Then $\varphi$ satisfies condition $\left(\mathbf{e n g}_{H}\right)_{K}$ if and only if $\varphi$ satisfies condition $\left(\mathbf{e n g}_{\mathbb{H}^{n}}\right)_{K}$.

Proof If $\varphi$ satisfies $\left(\mathbf{e n g}_{\mathbb{H}^{n}}\right)_{K}$, it is clear that $\left(\mathbf{e n g}_{H}\right)_{K}$ holds. Let us prove the converse. Take any $\xi^{\prime} \in \mathbb{S}_{\varphi}^{\mathbb{H}^{n}}(\xi, p, s)$, i.e. $\xi^{\prime}=\xi \circ \exp \left(v_{1}\right) \circ \exp \left(v_{2}\right) \circ \exp \left(v_{3}\right)$, with $v_{i} \in V_{1}$ and with

$$
\begin{aligned}
& \xi_{1}:=\xi \circ \exp \left(v_{1}\right) \in S_{\varphi}^{H}(\xi, p, s), \\
& \xi_{2}:=\xi \circ \exp \left(v_{1}\right) \circ \exp \left(v_{2}\right) \in S_{\varphi}^{H}\left(\xi_{1}, p_{1}, s\right), \quad \text { with } p_{1} \in \partial_{H} \varphi\left(\xi_{1}\right), \\
& \xi^{\prime} \in S_{\varphi}^{H}\left(\xi_{2}, p_{2}, s\right), \quad \text { with } p_{2} \in \partial_{H} \varphi\left(\xi_{2}\right)
\end{aligned}
$$

we have to show that $\xi \in \mathbb{S}_{\varphi}^{\mathbb{H}^{n}}\left(\xi^{\prime}, q, K^{\prime} s\right)$, for every $q \in \partial_{H} \varphi\left(\xi^{\prime}\right)$. The assumption implies

$$
\begin{array}{ll}
\xi \in S_{\varphi}^{H}\left(\xi_{1}, p_{1}, K^{\prime} s\right), & \forall p_{1} \in \partial_{H} \varphi\left(\xi_{1}\right), \\
\xi_{1} \in S_{\varphi}^{H}\left(\xi_{2}, p_{2}, K^{\prime} s\right), & \forall p_{2} \in \partial_{H} \varphi\left(\xi_{2}\right), \\
\xi_{2} \in S_{\varphi}^{H}\left(\xi^{\prime}, q, K^{\prime} s\right), & \forall q \in \partial_{H} \varphi\left(\xi^{\prime}\right) .
\end{array}
$$

Hence, for every $q \in \partial_{H} \varphi\left(\xi^{\prime}\right)$,

$$
\begin{gathered}
\xi \in \underset{\xi_{2} \in S_{\varphi}^{H}\left(\xi^{\prime}, q, K^{\prime} s\right), p_{2} \in \partial_{H} \varphi\left(\xi_{2}\right),}{\bigcup_{\varphi}^{H}\left(\xi_{1}, p_{1}, K^{\prime} s\right)=\mathbb{S}_{\varphi}^{\mathbb{H}^{n}}\left(\xi^{\prime}, q, K^{\prime} s\right) .} \\
\xi_{1} \in S_{\varphi}^{H}\left(\xi_{2}, p_{2}, K^{\prime} s\right), p_{1} \in \partial_{H} \varphi\left(\xi_{1}\right)
\end{gathered}
$$

Clearly, if $\varphi$ is a strictly $H$-convex function satisfying the engulfing property $E\left(\mathbb{H}^{n}, K\right)$, then Remark 5.1, Proposition 5.2 and Proposition 4.2 imply that $\varphi$ is $H$-differentiable.

The next result will be crucial to our purposes:

Proposition 5.3 Let $\varphi: \mathbb{H}^{n} \rightarrow \mathbb{R}$ be an H-differentiable and strictly $H$-convex function. Then, for every $\xi \in \mathbb{H}^{n}, r>0$, we have

$$
\begin{aligned}
& \bigcup S_{\varphi}^{H}\left(\xi_{2}, m_{\varphi}^{H}\left(\xi_{2}, r\right)\right) \subset \widetilde{B}(\xi, r) \\
& \xi_{1} \in S_{\varphi}^{H}\left(\xi, m_{\varphi}^{H}(\xi, r)\right) \\
& \xi_{2} \in S_{\varphi}^{H}\left(\xi_{1}, m_{\varphi}^{H}\left(\xi_{1}, r\right)\right) \\
& \subset \quad \bigcup S_{\varphi}^{H}\left(\xi_{2}, M_{\varphi}^{H}\left(\xi_{2}, r\right)\right) \text {. } \\
& \xi_{1} \in S_{\varphi}^{H}\left(\xi, M_{\varphi}^{H}(\xi, r)\right) \\
& \xi_{2} \in S_{\varphi}^{H}\left(\xi_{1}, M_{\varphi}^{H}\left(\xi_{1}, r\right)\right)
\end{aligned}
$$

Let us emphasize that, despite its appearance, the first set in (5.8) is not an $\mathbb{H}^{n}$ section, since $m_{\varphi}^{H}\left(\xi_{1}, r\right)$, for $\xi_{1} \in S_{\varphi}^{H}\left(\xi, m_{\varphi}^{H}(\xi, r)\right)$, and $m_{\varphi}^{H}\left(\xi_{2}, r\right)$, for $\xi_{2} \in$ $S_{\varphi}^{H}\left(\xi_{1}, m_{\varphi}^{H}\left(\xi_{1}, r\right)\right)$, are not fixed values. A similar comment holds for the set in (5.9). 
Proof of Proposition 5.3 By the inclusions in (3.6), we easily have

$$
\begin{aligned}
& \bigcup S_{\varphi}^{H}\left(\xi_{2}, m_{\varphi}^{H}\left(\xi_{2}, r\right)\right) \subset \quad \bigcup B_{g}\left(\xi_{2}, r\right) \cap H_{\xi_{2}} \\
& \xi_{1} \in S_{\varphi}^{H}\left(\xi, m_{\varphi}^{H}(\xi, r)\right) \\
& \xi_{1} \in S_{\varphi}^{H}\left(\xi, m_{\varphi}^{H}(\xi, r)\right) \\
& \xi_{2} \in S_{\varphi}^{H}\left(\xi_{1}, m_{\varphi}^{H}\left(\xi_{1}, r\right)\right) \\
& \xi_{2} \in S_{\varphi}^{H}\left(\xi_{1}, m_{\varphi}^{H}\left(\xi_{1}, r\right)\right) \\
& \subset \quad \bigcup B_{g}\left(\xi_{2}, r\right) \cap H_{\xi_{2}} \\
& \xi_{1} \in B_{g}(\xi, r) \cap H_{\xi} \\
& \xi_{2} \in B_{g}\left(\xi_{1}, r\right) \cap H_{\xi_{1}} \\
& \subset \quad \bigcup S_{\varphi}^{H}\left(\xi_{2}, M_{\varphi}^{H}\left(\xi_{2}, r\right)\right) \\
& \xi_{1} \in B_{g}(\xi, r) \cap H_{\xi} \\
& \xi_{2} \in B_{g}\left(\xi_{1}, r\right) \cap H_{\xi_{1}} \\
& \subset \quad \bigcup S_{\varphi}^{H}\left(\xi_{2}, M_{\varphi}^{H}\left(\xi_{2}, r\right)\right) \\
& \xi_{1} \in S_{\varphi}^{H}\left(\xi, M_{\varphi}^{H}(\xi, r)\right) \\
& \xi_{2} \in S_{\varphi}^{H}\left(\xi_{1}, M_{\varphi}^{H}\left(\xi_{1}, r\right)\right)
\end{aligned}
$$

for every $\xi \in \mathbb{H}^{n}, r>0$. Hence the assertion holds.

In order to prove our main result concerning the engulfing property of the $\mathbb{H}^{n}$ sections, an extension to the Heisenberg case of the inequalities (2.8), (2.9) and (2.11) turns out to be quite useful:

Proposition 5.4 Let $\varphi$ be a strictly $H$-convex function in $E(H, K)$. Then, for every $r \geq 0$ and $\xi \in \mathbb{H}^{n}$, we have

$$
\begin{aligned}
& M_{\varphi}^{H}(\xi, 2 r) \leq B_{1} M_{\varphi}^{H}(\xi, r), \\
& m_{\varphi}^{H}(\xi, 2 r) \leq B_{2} m_{\varphi}^{H}(\xi, r), \\
& B_{4} m_{\varphi}^{H}(\xi, r) \leq m_{\varphi}^{H}(\xi, 2 r),
\end{aligned}
$$

where $B_{1}, B_{2}$ and $B_{4}$ depend only on $K$, and $B_{i}>1$.

Proof Proposition 4.2 implies that $\varphi$ is $H$-differentiable and, if we consider its restriction to any horizontal segment, we obtain a strictly convex and differentiable function. To be precise, for every $\xi \in \mathbb{H}^{n}$ and $v \in V_{1}$ with $\|v\|=1$ the function $\widehat{\varphi}_{\xi, v}: \mathbb{R} \rightarrow \mathbb{R}$, defined as in (3.3), satisfies condition ii. in Th. 2.1. By (2.8) in Remark 2.1 we obtain

$$
M_{\widehat{\varphi}_{\xi, v}}(0,2 r) \leq B_{1} M_{\widehat{\varphi}_{\xi}, v}(0, r),
$$

where $B_{1}$ depends only on $K$. Hence we have

$$
\begin{aligned}
& \max _{\left\{w \in V_{1}: w= \pm 2 r v\right\}}\left(\varphi(\xi \circ \exp w)-\varphi(\xi)-\nabla_{H} \varphi(\xi) \cdot w\right) \leq \\
& \leq B_{1} \max _{\left\{w \in V_{1}: w= \pm r v\right\}}\left(\varphi(\xi \circ \exp w)-\varphi(\xi)-\nabla_{H} \varphi(\xi) \cdot w\right) ;
\end{aligned}
$$


taking the maximum w.r.t. to $v$, with $\|v\|=1$, we obtain (5.10).

A similar proof, via inequality (2.9) in Remark 2.1 and inequality (2.11) in Proposition 2.1, shows (5.11) and (5.12), respectively.

In the final part of this section we will prove our main result concerning the relationship between round $H$-sections and the engulfing property of the $\mathbb{H}^{n}$-sections. The proof will be quite technical, deserving a few previous estimates.

Let $\varphi: \mathbb{H}^{n} \rightarrow \mathbb{R}$ be an $H$-convex function with round $H$-sections (with constant $\left.K_{0}\right)$. Then, $\varphi \in E(H, K)$, and has controlled $H$-slope (with constant $K_{1}$ ), where both $K, K_{1}$ depend on $K_{0}$. Denote by $\gamma$ any positive integer such that

$$
K_{1} \leq B_{4}^{\gamma}
$$

Thus, from (3.7), and by iterating inequality (5.12), we obtain

$$
M_{\varphi}^{H}(\xi, r) \leq K_{1} m_{\varphi}^{H}(\xi, r) \leq \frac{K_{1}}{B_{4}^{\gamma}} m_{\varphi}^{H}\left(\xi, 2^{\gamma} r\right) \leq m_{\varphi}^{H}\left(\xi, 2^{\gamma} r\right)
$$

Then, we have that

$$
M_{\varphi}^{H}(\xi, r) \leq m_{\varphi}^{H}\left(\xi, 2^{\gamma} r\right),
$$

for every $r>0$ and $\xi \in \mathbb{H}^{n}$, where $\gamma>1$ depends only on $K_{0}$ in (3.4).

The next proposition holds:

Proposition 5.5 Let $\varphi: \mathbb{H}^{n} \rightarrow \mathbb{R}$ be a function with round $H$-sections (with $K_{0}$ as in (3.4)). Then, there exists a constant $C_{1}>0$ such that, if $\xi^{\prime} \in S_{\varphi}^{H}(\xi, s)$, then

$$
m_{\varphi}^{H}\left(\xi^{\prime}, r\right) \leq C_{1} m_{\varphi}^{H}(\xi, r)
$$

for $r$ such that $s=m_{\varphi}^{H}(\xi, r)$. The constant $C_{1}$ depends only on $K_{0}$.

Proof Since $\varphi$ has round $H$-sections, it is strictly $H$-convex, $H$-differentiable and it satisfies the engulfing property $E(H, K)$, where $K$ depends only on $K_{0}$. Let $\xi^{\prime}=$ $\xi \circ \exp v \in S_{\varphi}^{H}(\xi, s)$, and set $r$ such that $s=m_{\varphi}^{H}(\xi, r)$ : clearly,

$$
\xi^{\prime} \in S_{\varphi}^{H}\left(\xi, m_{\varphi}^{H}(\xi, r)\right) \subset S_{\varphi}^{H}\left(\xi, M_{\varphi}^{H}(\xi, r)\right) \subset S_{\varphi}^{H}\left(\xi, M_{\varphi}^{H}(\xi, 2 r)\right) .
$$

Moreover, since $\xi^{\prime} \in S_{\varphi}^{H}\left(\xi, m_{\varphi}^{H}(\xi, r)\right)$, by (3.6) we have that

$$
\xi^{\prime} \circ \exp ( \pm r v /\|v\|) \in B_{g}(\xi, 2 r) \cap H_{\xi} \subset S_{\varphi}^{H}\left(\xi, M_{\varphi}^{H}(\xi, 2 r)\right)
$$

Furthermore, since $\varphi \in E(H, K)$, we have that $\xi^{\prime} \in S_{\varphi}^{H}\left(\xi, M_{\varphi}^{H}(\xi, 2 r)\right)$ gives

$$
S_{\varphi}^{H}\left(\xi, M_{\varphi}^{H}(\xi, 2 r)\right) \cap H_{\xi^{\prime}} \subset S_{\varphi}^{H}\left(\xi^{\prime}, K^{\prime} M_{\varphi}^{H}(\xi, 2 r)\right) \cap H_{\xi} .
$$


This implies $\xi^{\prime} \circ \exp ( \pm r v /\|v\|) \in S_{\varphi}^{H}\left(\xi^{\prime}, K M_{\varphi}^{H}(\xi, 2 r)\right)$. Now, by (5.10) and (3.4), we have

$$
\begin{aligned}
\xi^{\prime} \circ \exp ( \pm r v /\|v\|) \in S_{\varphi}^{H}\left(\xi^{\prime}, K M_{\varphi}^{H}(\xi, 2 r)\right) & \subset S_{\varphi}^{H}\left(\xi^{\prime}, K B_{1} M_{\varphi}^{H}(\xi, r)\right) \\
& \subset S_{\varphi}^{H}\left(\xi^{\prime}, K B_{1} K_{0} m_{\varphi}^{H}(\xi, r)\right)
\end{aligned}
$$

where $B_{1}$ depends only on $K_{0}$. Then,

$$
\begin{aligned}
m_{\varphi}^{H}\left(\xi^{\prime}, r\right) & \leq \varphi\left(\xi^{\prime} \circ \exp ( \pm r v /\|v\|)\right)-\varphi\left(\xi^{\prime}\right)-\nabla_{H} \varphi\left(\xi^{\prime}\right) \cdot( \pm r v /\|v\|) \\
& \leq K B_{1} K_{0} m_{\varphi}^{H}(\xi, r)
\end{aligned}
$$

A result similar to Proposition 5.5, involving now the $\mathbb{H}^{n}$-sections $\mathbb{S}_{\varphi}^{\mathbb{H}^{n}}(\xi, s)$, holds, but the proof is much more delicate:

Proposition 5.6 Let $\varphi: \mathbb{H}^{n} \rightarrow \mathbb{R}$ be a function with round $H$-sections (with $K_{0}$ as in (3.4)). Then, there exists a constant $B_{5}>0$ such that, if $\xi^{\prime} \in \mathbb{S}_{\varphi}^{\mathbb{H}^{n}}(\xi, s)$, then

$$
m_{\varphi}^{H}\left(\xi^{\prime}, r\right) \leq B_{5} m_{\varphi}^{H}(\xi, r)
$$

for $r$ such that $s=m_{\varphi}^{H}(\xi, r)$. The constant $B_{5}$ depends only on $K_{0}$.

Proof Since $\varphi$ has round $H$-sections, it belongs to $E(H, K)$, where $K$ depends only on $K_{0}$, and for every $r \geq 0$ and $\xi \in \mathbb{H}^{n}$ the inequality (5.12) holds. In addition, by Proposition 3.3, the function $m_{\varphi}^{H}(\xi, \cdot):[0,+\infty) \rightarrow[0,+\infty)$ is invertible.

Take any $\xi_{3} \in \mathbb{S}_{\varphi}^{\mathbb{H}^{n}}\left(\xi_{0}, s\right)$, i.e. $\xi_{1} \in S_{\varphi}^{H}\left(\xi_{0}, s\right), \xi_{2} \in S_{\varphi}^{H}\left(\xi_{1}, s\right)$ and $\xi_{3} \in S_{\varphi}^{H}\left(\xi_{2}, s\right)$, with $s$ such that $s=m_{\varphi}^{H}\left(\xi_{0}, r\right)$. By Proposition 5.5 and $\xi_{1} \in S_{\varphi}^{H}\left(\xi_{0}, s\right)$, we have

$$
\left(m_{\varphi}^{H}\left(\xi_{0}, \cdot\right)\right)^{-1}(s) \leq\left(m_{\varphi}^{H}\left(\xi_{1}, \cdot\right)\right)^{-1}\left(C_{1} s\right)
$$

Similarly, since $\xi_{2} \in S_{\varphi}^{H}\left(\xi_{1}, s\right)$, we have

$$
\left(m_{\varphi}^{H}\left(\xi_{1}, \cdot\right)\right)^{-1}(s) \leq\left(m_{\varphi}^{H}\left(\xi_{2}, \cdot\right)\right)^{-1}\left(C_{1} s\right)
$$

Let us prove that there exists a constant $C$, which depends only on $C_{1}$ and $B_{4}$, and hence on $K_{0}$, such that

$$
\left(m_{\varphi}^{H}(\xi, \cdot)\right)^{-1}\left(C_{1} s\right) \leq C\left(m_{\varphi}^{H}(\xi, \cdot)\right)^{-1}(s), \quad \forall \xi \in \mathbb{H}^{n} .
$$

Inequality (5.12) is equivalent to

$$
\left(m_{\varphi}^{H}(\xi, \cdot)\right)^{-1}\left(B_{4} \tilde{s}\right) \leq 2\left(m_{\varphi}^{H}(\xi, \cdot)\right)^{-1}(\tilde{s}), \quad \forall \tilde{s} \geq 0 ;
$$


by choosing $\beta \in \mathbb{N}$ such that $C_{1} \leq B_{4}^{\beta}$, iterating the previous inequality and taking into account that $\tilde{s} \mapsto\left(m_{\varphi}^{H}(\xi, \cdot)\right)^{-1}(\tilde{s})$ is an increasing function we obtain

$$
\left(m_{\varphi}^{H}(\xi, \cdot)\right)^{-1}\left(C_{1} s\right) \leq 2^{\beta}\left(m_{\varphi}^{H}(\xi, \cdot)\right)^{-1}\left(\frac{C_{1} s}{B_{4}^{\beta}}\right) \leq 2^{\beta}\left(m_{\varphi}^{H}(\xi, \cdot)\right)^{-1}(s) .
$$

Hence, (5.18) holds with $C=2^{\beta}$; now, by (5.16), (5.19) and (5.17), we obtain

$$
\begin{aligned}
\left(m_{\varphi}^{H}\left(\xi_{0}, \cdot\right)\right)^{-1}(s) & \leq\left(m_{\varphi}^{H}\left(\xi_{1}, \cdot\right)\right)^{-1}\left(C_{1} s\right) \\
& \leq 2^{\beta}\left(m_{\varphi}^{H}\left(\xi_{1}, \cdot\right)\right)^{-1}(s) \\
& \leq 2^{\beta}\left(m_{\varphi}^{H}\left(\xi_{2}, \cdot\right)\right)^{-1}\left(C_{1} s\right) .
\end{aligned}
$$

A similar argument proves that $\xi_{3} \in S_{\varphi}^{H}\left(\xi_{2}, s\right)$ implies

$$
\left(m_{\varphi}^{H}\left(\xi_{0}, \cdot\right)\right)^{-1}(s) \leq 2^{2 \beta}\left(m_{\varphi}^{H}\left(\xi_{3}, \cdot\right)\right)^{-1}\left(C_{1} s\right)
$$

Now, recalling that $s=m_{\varphi}^{H}\left(\xi_{0}, r\right)$, the previous inequality gives

$$
m_{\varphi}^{H}\left(\xi_{3}, 2^{-2 \beta} r\right) \leq C_{1} m_{\varphi}^{H}\left(\xi_{0}, r\right)
$$

Finally, (5.11) and (5.20) implies

$$
m_{\varphi}^{H}\left(\xi_{3}, r\right) \leq B_{2}^{2 \beta} C_{1} m_{\varphi}^{H}\left(\xi_{3}, r\right) \leq B_{2}^{2 \beta} C_{1} m_{\varphi}^{H}\left(\xi_{0}, r\right)
$$

and the proof in finished.

In order to introduce and prove the main result of the section, we need the following

Lemma 5.1 Let $\varphi: \mathbb{H}^{n} \rightarrow \mathbb{R}$ be an $H$-convex function with round $H$-sections.

a. If $\xi^{\prime} \in S_{\varphi}^{H}\left(\xi, m_{\varphi}^{H}(\xi, r)\right)$ for some $r>0$, then

$$
m_{\varphi}^{H}(\xi, r) \leq m_{\varphi}^{H}\left(\xi^{\prime}, 2^{1+\gamma} r\right)
$$

with $\gamma$ as in (5.13);

b. if $\xi^{\prime} \in S_{\varphi}^{H}\left(\xi, M_{\varphi}^{H}(\xi, r)\right)$ for some $r>0$, then

$$
M_{\varphi}^{H}\left(\xi^{\prime}, r\right) \leq m_{\varphi}^{H}\left(\xi, 2^{2+3 \gamma+\tilde{\gamma}} r\right),
$$

with $\tilde{\gamma}$ as in (5.27) which depends only on $K_{0}$. 
Proof Let us consider $\xi^{\prime}=\xi \circ \exp v \in S_{\varphi}^{H}\left(\xi, m_{\varphi}^{H}(\xi, r)\right)$ : by (3.6) we have

$$
\|v\| \leq r
$$

The $H$-convexity of $\varphi$ and the $H$-monotonicity of $\nabla_{H} \varphi$ give

$$
\begin{aligned}
& \varphi(\xi)-\varphi(\xi \circ \exp v)-\nabla_{H} \varphi(\xi \circ \exp v) \cdot v \geq 0 \\
& \left(\nabla_{H} \varphi(\xi \circ \exp v)-\nabla_{H} \varphi(\xi)\right) \cdot v \frac{r}{\|v\|} \geq 0
\end{aligned}
$$

Again the $H$-convexity of $\varphi$ and (5.23)-(5.25) give

$$
\begin{aligned}
m_{\varphi}^{H}(\xi, r) \leq & \varphi\left(\xi \circ \exp \left(-\frac{v}{\|v\|} r\right)\right)-\varphi(\xi)-\nabla_{H} \varphi(\xi) \cdot\left(-\frac{v}{\|v\|} r\right) \\
\leq & \varphi\left(\xi \circ \exp \left(-\frac{v}{\|v\|} r\right)\right)-\varphi(\xi \circ \exp v) \\
& -\nabla_{H} \varphi(\xi \circ \exp v) \cdot\left(-v \frac{r}{\|v\|}-v\right) \\
\leq & M_{\varphi}^{H}\left(\xi^{\prime}, 2 r\right) .
\end{aligned}
$$

Therefore (5.21) follows from (5.14).

Let us prove b. Take any $\xi^{\prime} \in S_{\varphi}^{H}\left(\xi, M_{\varphi}^{H}(\xi, r)\right)$; since $\varphi \in E(H, K)$, with $K$ depending on $K_{0}$ only (see Theorem 4.1), then $\xi \in S_{\varphi}^{H}\left(\xi^{\prime}, K M_{\varphi}^{H}(\xi, r)\right)$. From (5.14) we have $\xi^{\prime} \in S_{\varphi}^{H}\left(\xi, M_{\varphi}^{H}(\xi, r)\right) \subset S_{\varphi}^{H}\left(\xi, m_{\varphi}^{H}\left(\xi, 2^{\gamma} r\right)\right)$, and (5.21) implies that

$$
K M_{\varphi}^{H}(\xi, r) \leq K m_{\varphi}^{H}\left(\xi, 2^{\gamma} r\right) \leq K m_{\varphi}^{H}\left(\xi^{\prime}, 2^{2 \gamma+1} r\right) .
$$

Now, let $\tilde{\gamma} \in \mathbb{N}$ be such that

$$
K \leq B_{4}^{\tilde{\gamma}} .
$$

By iterating inequality (5.12) and (5.14), inequality (5.26) gives

$$
\begin{aligned}
K M_{\varphi}^{H}(\xi, r) & \leq K m_{\varphi}^{H}\left(\xi^{\prime}, 2^{2 \gamma+1} r\right) \leq \frac{K}{B_{4}^{\tilde{\gamma}}} m_{\varphi}^{H}\left(\xi^{\prime}, 2^{1+2 \gamma+\tilde{\gamma}} r\right) \\
& \leq m_{\varphi}^{H}\left(\xi^{\prime}, 2^{1+2 \gamma+\tilde{\gamma}} r\right) .
\end{aligned}
$$

Hence, $\xi \in S_{\varphi}^{H}\left(\xi^{\prime}, K M_{\varphi}^{H}(\xi, r)\right) \subset S_{\varphi}^{H}\left(\xi^{\prime}, m_{\varphi}^{H}\left(\xi^{\prime}, 2^{1+2 \gamma+\tilde{\gamma}} r\right)\right)$. Finally, the inequalities (5.14) and (5.21) imply

$$
M_{\varphi}^{H}\left(\xi^{\prime}, r\right) \leq m_{\varphi}^{H}\left(\xi^{\prime}, 2^{\gamma} r\right) \leq m_{\varphi}^{H}\left(\xi^{\prime}, 2^{1+2 \gamma+\tilde{\gamma}} r\right) \leq m_{\varphi}^{H}\left(\xi, 2^{2+3 \gamma+\tilde{\gamma}} r\right)
$$

We are now in the position to prove the first part of our main result in Theorem 1.1: 
Proof of Theorem 1.1 i Let $\varphi: \mathbb{H}^{n} \rightarrow \mathbb{R}$ be an $H$-convex function with round $H$ sections. Let us prove that $\varphi$ satisfies the engulfing property $E\left(\mathbb{H}^{n}, K\right)$. Fix $\xi \in \mathbb{H}^{n}$ and $s>0$. Let us suppose that $\xi^{\prime} \in \mathbb{S}_{\varphi}^{\mathbb{H}^{n}}(\xi, s)$ : we have to prove that $\mathbb{S}_{\varphi}^{\mathbb{H}^{n}}(\xi, s) \subset$ $\mathbb{S}_{\varphi}^{\mathbb{H}^{n}}\left(\xi^{\prime}, K s\right)$, where $K$ is a constant which depends only on $K_{0}$ in (3.4).

Let $r$ be such that $s=m_{\varphi}^{H}(\xi, r)$. By definition,

$$
\begin{aligned}
\mathbb{S}_{\varphi}^{\mathbb{H}^{n}}(\xi, s)= & \bigcup S_{\varphi}^{H}\left(\xi_{2}, m_{\varphi}^{H}(\xi, r)\right) . \\
& \xi_{1} \in S_{\varphi}^{H}\left(\xi, m_{\varphi}^{H}(\xi, r)\right) \\
& \xi_{2} \in S_{\varphi}^{H}\left(\xi_{1}, m_{\varphi}^{H}(\xi, r)\right)
\end{aligned}
$$

For every $\xi_{1} \in S_{\varphi}^{H}\left(\xi, m_{\varphi}^{H}(\xi, r)\right)$, by applying a. in Lemma 5.1, we get

$$
m_{\varphi}^{H}(\xi, r) \leq m_{\varphi}^{H}\left(\xi_{1}, 2^{1+\gamma} r\right)
$$

and, by (5.29), we get

$$
\mathbb{S}_{\varphi}^{\mathbb{H}^{n}}(\xi, s) \subset \underbrace{\left.\bigcup_{\varphi}^{H}\left(\xi_{1}, 2^{1+\gamma} r\right)\right)}_{\substack{\xi_{1} \in S_{\varphi}^{H} \\ \xi_{2} \in S_{\varphi}^{H}\left(\xi_{1}, m_{\varphi}^{H}(\xi, r)\right)}}
$$

For every $\xi_{2} \in S_{\varphi}^{H}\left(\xi_{1}, m_{\varphi}^{H}\left(\xi_{1}, 2^{1+\gamma} r\right)\right)$ with $\xi_{1} \in S_{\varphi}^{H}\left(\xi, m_{\varphi}^{H}(\xi, r)\right)$, via a. in Lemma 5.1 we get

$$
m_{\varphi}^{H}\left(\xi_{1}, 2^{1+\gamma} r\right) \leq m_{\varphi}^{H}\left(\xi_{2}, 2^{2+2 \gamma} r\right) .
$$

Using now (5.30) and (5.32), relation (5.31) becomes

$$
\mathbb{S}_{\varphi}^{\mathbb{H}^{H^{n}}}(\xi, s) \subset \underbrace{\left.\bigcup_{\varphi}^{H}\left(\xi_{1}, 2^{1+\gamma} r\right)\right)}_{\substack{\xi_{1} \in S_{\varphi}^{H} \\ \xi_{2} \in S_{\varphi}^{H}\left(\xi_{1}, m_{\varphi}^{H}(\xi, r)\right)}}
$$

Since $\gamma>0$, we have the following inclusions:

$$
\begin{aligned}
\mathbb{S}_{\varphi}^{\mathbb{H}^{n}}(\xi, s) \subset & \bigcup_{\xi_{1} \in S_{\varphi}^{H}\left(\xi, m_{\varphi}^{H}\left(\xi, 2^{2+2 \gamma} r\right)\right)} S_{\varphi}^{H}\left(\xi_{2}, m_{\varphi}^{H}\left(\xi_{2}, 2^{2+2 \gamma} r\right)\right) \\
& \xi_{2} \in S_{\varphi}^{H}\left(\xi_{1}, m_{\varphi}^{H}\left(\xi_{1}, 2^{2+2 \gamma} r\right)\right) \\
\subset & \widetilde{B}\left(\xi, 2^{2+2 \gamma} r\right),
\end{aligned}
$$

where the last inclusion comes from (5.8). Then, using the inclusions in (5.3), we get

$$
\begin{aligned}
\mathbb{S}_{\varphi}^{\mathbb{H}^{n}}(\xi, s) & \subset \widetilde{B}\left(\xi, 2^{2+2 \gamma} r\right) \subset B_{g}\left(\xi, 32^{2+2 \gamma} r\right) n \\
& \subset B_{g}\left(\xi^{\prime}, 32^{3+2 \gamma} r\right) \subset \widetilde{B}\left(\xi^{\prime}, 3 C 2^{3+2 \gamma} r\right),
\end{aligned}
$$


where $C$ is the constant in the Folland-Stein Lemma. Inclusions (5.9) and (5.34) give

$$
\mathbb{S}_{\varphi}^{\mathbb{H}^{n}}(\xi, s) \subset \underbrace{\left.\left.\bigcup \xi_{3}, 3 C 2^{3+2 \gamma} r\right)\right)}_{\substack{\left.\xi_{3} \in S_{\varphi}^{H} \\ \xi_{4} \in \xi_{\varphi}^{\prime}, M_{\varphi}^{H}\left(\xi_{3}, M_{\varphi}^{H}, 3 C 2^{3+2 \gamma} r\right)\right)}}
$$

Now, applying twice (5.22) in Lemma 5.1, we have, by the previous inclusion,

$$
\begin{aligned}
& \mathbb{S}_{\varphi}^{\mathbb{H}^{n}}(\xi, s) \subset \quad \bigcup S_{\varphi}^{H}\left(\xi_{4}, M_{\varphi}^{H}\left(\xi_{3}, 3 C 2^{5+5 \gamma+\tilde{\gamma}_{r}} r\right)\right. \\
& \xi_{3} \in S_{\varphi}^{H}\left(\xi^{\prime}, M_{\varphi}^{H}\left(\xi^{\prime}, 3 C 2^{3+2 \gamma_{r}}\right)\right) \\
& \xi_{4} \in S_{\varphi}^{H}\left(\xi_{3}, M_{\varphi}^{H}\left(\xi_{3}, 3 C 2^{3+2 \gamma} r\right)\right) \\
& \subset \quad \bigcup S_{\varphi}^{H}\left(\xi_{4}, M_{\varphi}^{H}\left(\xi_{3}, 3 C 2^{5+5 \gamma+\tilde{\gamma}} r\right)\right) \\
& \xi_{3} \in S_{\varphi}^{H}\left(\xi^{\prime}, M_{\varphi}^{H}\left(\xi^{\prime}, 3 C 2^{5+5 \gamma+\tilde{\gamma}_{r}} r\right)\right. \\
& \xi_{4} \in S_{\varphi}^{H}\left(\xi_{3}, M_{\varphi}^{H}\left(\xi_{3}, 3 C 2^{5+5 \gamma+\tilde{\gamma}} r\right)\right) \\
& \subset \quad \bigcup \quad S_{\varphi}^{H}\left(\xi_{4}, M_{\varphi}^{H}\left(\xi^{\prime}, 3 C 2^{7+8 \gamma+2 \tilde{\gamma}} r\right)\right) \\
& \xi_{3} \in S_{\varphi}^{H}\left(\xi^{\prime}, M_{\varphi}^{H}\left(\xi^{\prime}, 3 C 2^{5+5 \gamma+\tilde{\gamma}} r\right)\right) \\
& \xi_{4} \in S_{\varphi}^{H}\left(\xi_{3}, M_{\varphi}^{H}\left(\xi^{\prime}, 3 C 2^{7+8 \gamma+2 \tilde{\gamma}_{r}}\right)\right) \\
& \subset \quad \bigcup S_{\varphi}^{H}\left(\xi_{4}, M_{\varphi}^{H}\left(\xi^{\prime}, 3 C 2^{7+8 \gamma+2 \tilde{\gamma}} r\right)\right) \\
& \xi_{3} \in S_{\varphi}^{H}\left(\xi^{\prime}, M_{\varphi}^{H}\left(\xi^{\prime}, 3 C 2^{7+8 \gamma+2 \tilde{\gamma}} r\right)\right) \\
& \xi_{4} \in S_{\varphi}^{H}\left(\xi_{3}, M_{\varphi}^{H}\left(\xi^{\prime}, 3 C 2^{7+8 \gamma+2 \tilde{\gamma}} r\right)\right) \\
& =\mathbb{S}_{\varphi}^{\mathbb{H}^{n}}\left(\xi^{\prime}, M_{\varphi}^{H}\left(\xi^{\prime}, 3 C 2^{7+8 \gamma+2 \tilde{\gamma}} r\right)\right) \text {. }
\end{aligned}
$$

Set $\tilde{C}=3 C 2^{7+8 \gamma+2 \tilde{\gamma}}$, and take any $\delta \in \mathbb{N}$ such that $\tilde{C} \leq 2^{\delta}$; clearly, both $\tilde{C}$ and $\delta$ they depend only on $K_{0}$. Hence, we have the following inclusions:

$$
\begin{aligned}
\mathbb{S}_{\varphi}^{\mathbb{H}^{n^{n}}}(\xi, s) & \subset \mathbb{S}_{\varphi}^{\mathbb{H}^{n}}\left(\xi^{\prime}, M_{\varphi}^{H}\left(\xi^{\prime}, \tilde{C} r\right)\right) \\
& \subset \mathbb{S}_{\varphi}^{\mathbb{H}^{n}}\left(\xi^{\prime}, B_{1}^{\delta} M_{\varphi}^{H}\left(\xi^{\prime}, \tilde{C} 2^{-\delta} r\right)\right) \\
& \subset \mathbb{S}_{\varphi}^{\mathbb{H}^{n}}\left(\xi^{\prime}, B_{1}^{\delta} M_{\varphi}^{H}\left(\xi^{\prime}, r\right)\right) \\
& \subset \mathbb{S}_{\varphi}^{\mathbb{H}^{n}}\left(\xi^{\prime}, K_{1} B_{1}^{\delta} m_{\varphi}^{H}\left(\xi^{\prime}, r\right)\right) \\
& \subset \mathbb{S}_{\varphi}^{\mathbb{H}^{n}}\left(\xi^{\prime}, B_{5} K_{1} B_{1}^{\delta} m_{\varphi}^{H}(\xi, r)\right) \\
& \subset \mathbb{S}_{\varphi}^{\mathbb{H}^{n}}\left(\xi^{\prime}, B_{5} K_{1} B_{1}^{a} s\right) .
\end{aligned}
$$




\section{Balls and Quasi-distances via the $\mathbb{H}^{n}$-Sections of $H$-Convex Functions}

It is known that there is a deep connection between the existence of a quasi-distance $d$ on a given set $X$ and the existence of a family of subsets $\{S(x, s)\}_{\{x \in X, s>0\}}$ enjoying the following properties

$\left(P_{1}\right) \bigcap_{s>0} S(x, s)=\{x\}$, for every $x \in X$;

$\left(P_{2}\right) \bigcup_{s>0} S(x, s)=X$, for every $x \in X$;

$\left(P_{3}\right)$ for each $x \in X, s \mapsto S(x, s)$ is a non decreasing map;

$\left(P_{4}\right)$ there exists a constant $H$ such that, for all $y \in S(x, s)$,

$$
\begin{aligned}
& S(x, s) \subset S(y, H s), \\
& S(y, s) \subset S(x, H s) .
\end{aligned}
$$

As a matter of fact, the following result holds:

Lemma 6.1 (see Lemma 1 in [1]) Let $X$ be a set and $S: X \times \mathbb{R}^{+} \rightarrow \mathcal{P}(X)$ be a set-valued map such that the family $\{S(x, s)\}$ has the properties $\left(P_{1}\right)-\left(P_{4}\right)$. Then, the function $d: X \times X \rightarrow[0,+\infty)$ defined by

$$
d(x, y)=\inf \{s: x \in S(y, s), y \in S(x, s)\}
$$

is a quasi-distance. On the other hand, given a quasi-distance $d$ defined on $X$, the family of the $d$-balls in $X$ satisfies the properties $\left(P_{1}\right)-\left(P_{4}\right)$.

In particular, in [1] the authors prove that the sections $S_{u}(x, r)$ of a convex function $u: \mathbb{R}^{k} \rightarrow \mathbb{R}$ satisfying the engulfing property, generate a quasi-distance.

Let us now consider an $H$-convex function $\varphi: \mathbb{H}^{n} \rightarrow \mathbb{R}$ with round $H$-sections; by taking all $s>0$ and $\xi \in \mathbb{H}^{n}=\mathbb{R}^{2 n+1}$ we obtain a family of sets $\left\{\mathbb{S}_{\varphi}^{\mathbb{H}}(\xi, s)\right\}_{\left\{\xi \in \mathbb{H}^{n}, s>0\right\}}$ (the $\mathbb{H}^{n}$-sections) for which conditions $\left(P_{1}\right)$ - $\left(P_{3}\right)$ trivially hold; moreover, due to Theorem 1.1, such family satisfies the engulfing property $E\left(\mathbb{H}^{n}, K\right)$, i.e. condition (6.1).

The next result shows that the family of $\mathbb{H}^{n}$-sections satisfies condition (6.2) too:

Theorem 6.1 Let $\varphi: \mathbb{H}^{n} \rightarrow \mathbb{R}$ be an $H$-convex function with round $H$-sections. Then, there exists a constant $\tilde{K}$, which depends only on $K_{0}$, such that, if $\xi^{\prime} \in \mathbb{S}_{\varphi}^{\mathbb{H}^{n}}(\xi, s)$, then $\mathbb{S}_{\varphi}^{\mathbb{H}^{n}}\left(\xi^{\prime}, s\right) \subset \mathbb{S}_{\varphi}^{\mathbb{H}^{n}}(\xi, \tilde{K} s)$.

Proof The proof follows the ideas in the proof of Theorem 1.1. Fix $\xi \in \mathbb{H}^{n} s>0$ and $\xi^{\prime} \in \mathbb{S}_{\varphi}^{\mathbb{H}^{n}}(\xi, s)$; let $r$ be such that $s=m_{\varphi}^{H}\left(\xi^{\prime}, r\right)$. Theorem 1.1 guarantees that $\varphi$ satisfies the engulfing property $E\left(\mathbb{H}^{n}, K\right)$, where $K$ depends only on $K_{0}$. Hence, $\xi \in \mathbb{S}_{\varphi}^{\mathbb{H}^{n}}\left(\xi^{\prime}, K s\right)$. Proposition 5.6 implies that

$$
m_{\varphi}^{H}(\xi, \widehat{r}) \leq B_{5} m_{\varphi}^{H}\left(\xi^{\prime}, \widehat{r}\right)
$$


for $\widehat{r}$ such that $K s=m_{\varphi}^{H}\left(\xi^{\prime}, \widehat{r}\right)$ (the constant $B_{5}$ depends only on $K_{0}$ ). Since, by Proposition 3.3, the function $r \mapsto m_{\varphi}^{H}(\xi, r)$ is an increasing function, we obtain

$$
m_{\varphi}^{H}(\xi, r) \leq B_{5} m_{\varphi}^{H}\left(\xi^{\prime}, \widehat{r}\right)=B_{5} K s
$$

By definition,

$$
\begin{aligned}
\mathbb{S}_{\varphi}^{\mathbb{H}^{n}}\left(\xi^{\prime}, s\right)= & \bigcup_{\xi_{1} \in S_{\varphi}^{H}}\left(\xi^{\prime}, m_{\varphi}^{H}\left(\xi^{\prime}, r\right)\right) \\
& \xi_{2} \in S_{\varphi}^{H}\left(\xi_{1}, m_{\varphi}^{H}\left(\xi^{\prime}, r\right)\right)
\end{aligned}
$$

Using exactly the same arguments as in the proof of Theorem 1.1, that allow us to pass from (5.29) to (5.33) (essentially, by exchanging the role of $\xi$ and $\xi^{\prime}$ ), we obtain

$$
\mathbb{S}_{\varphi}^{\mathbb{H}^{n}}\left(\xi^{\prime}, s\right) \subset \widetilde{B}\left(\xi^{\prime}, 2^{2+2 \gamma} r\right) \subset B_{g}\left(\xi^{\prime}, 32^{2+2 \gamma} r\right)
$$

Now, taking into account the definition of $\tilde{\gamma}$ in (5.27) and iterating inequality (5.12), we get

$$
K s=K m_{\varphi}^{H}\left(\xi^{\prime}, r\right) \leq \frac{K}{B_{4}^{\tilde{\gamma}}} m_{\varphi}^{H}\left(\xi^{\prime}, 2^{\tilde{\gamma}} r\right) \leq m_{\varphi}^{H}\left(\xi^{\prime}, 2^{\tilde{\gamma}} r\right)
$$

Since $\xi \in \mathbb{S}_{\varphi}^{\mathbb{H}^{n}}\left(\xi^{\prime}, K s\right)$, we obtain

$$
\mathbb{S}_{\varphi}^{\mathbb{H}^{n}}\left(\xi^{\prime}, K s\right) \subset \underset{\substack{\xi_{1} \in S_{\varphi}^{H}\left(\xi^{\prime}, m_{\varphi}^{H}\left(\xi^{\prime}, 2^{\tilde{\gamma}} r\right)\right) \\ \xi_{2} \in S_{\varphi}^{H}\left(\xi_{1}, m_{\varphi}^{H}\left(\xi^{\prime}, 2^{\tilde{\gamma}} r\right)\right)}}{\bigcup S_{\varphi}^{H}\left(\xi_{2}, m_{\varphi}^{H}\left(\xi^{\prime}, 2^{\tilde{\gamma}} r\right)\right) .}
$$

Using exactly the same arguments that allow us to pass from (6.4) to (6.5) (essentially, by exchanging the role of $r$ with $2^{\tilde{\gamma}} r$ ), we obtain

$$
\xi \in \widetilde{B}\left(\xi^{\prime}, 2^{2+2 \gamma+\tilde{\gamma}} r\right) \subset B_{g}\left(\xi^{\prime}, 32^{2+2 \gamma+\tilde{\gamma}} r\right) .
$$

Now, taking into account that $\tilde{\gamma}>0$, relations (6.5) and (6.8) give

$$
\mathbb{S}_{\varphi}^{\mathbb{H}^{n}}\left(\xi^{\prime}, s\right) \subset B_{g}\left(\xi^{\prime}, 32^{2+2 \gamma} r\right) \subset B_{g}\left(\xi, 32^{3+2 \gamma+\tilde{\gamma}} r\right) \subset \widetilde{B}\left(\xi, 3 C 2^{3+2 \gamma+\tilde{\gamma}} r\right)
$$

where $C$ in the previous inclusions is the constant in the Folland-Stein Lemma. Using the same arguments that allow us to pass from (5.34) to (5.35) (essentially, by replacing $3 C 2^{3+2 \gamma} r$ with $3 C 2^{3+2 \gamma+\tilde{\gamma}} r$ ), we obtain

$$
\mathbb{S}_{\varphi}^{\mathbb{H}^{n}}\left(\xi^{\prime}, s\right) \subset \mathbb{S}_{\varphi}^{\mathbb{H}^{n}}\left(\xi, M_{\varphi}^{H}\left(\xi, 3 C 2^{7+8 \gamma+3 \tilde{\gamma}} r\right)\right)
$$


Set $\widehat{C}=3 C 2^{7+8 \gamma+3 \tilde{\gamma}}$ and take $\widehat{\delta} \in \mathbb{N}$ such that $\widehat{C} \leq 2^{\widehat{\delta}}$; clearly, $\widehat{C}$ and $\widehat{\delta}$ depend only on $K_{0}$. We have the following inclusions:

$$
\begin{aligned}
\mathbb{S}_{\varphi}^{\mathbb{H}^{n}}\left(\xi^{\prime}, s\right) & \subset \mathbb{S}_{\varphi}^{\mathbb{H}^{n}}\left(\xi, M_{\varphi}^{H}(\xi, \widehat{C} r)\right) \\
& \subset \mathbb{S}_{\varphi}^{\mathbb{H}^{n}}\left(\xi, B_{1}^{\widehat{\delta}} M_{\varphi}^{H}\left(\xi, \widehat{C} 2^{-\widehat{\delta}} r\right)\right) \\
& \subset \mathbb{S}_{\varphi}^{\mathbb{H}^{n}}\left(\xi, B_{1}^{\widehat{\delta}} M_{\varphi}^{H}(\xi, r)\right) \\
& \subset \mathbb{S}_{\varphi}^{\mathbb{H}^{n}}\left(\xi, K_{1} B_{1}^{\widehat{\delta}} m_{\varphi}^{H}(\xi, r)\right) \\
& \subset \mathbb{S}_{\varphi}^{\mathbb{H}^{n}}\left(\xi, B_{5} K K_{1} B_{1}^{\widehat{\delta}} s\right)
\end{aligned}
$$

which concludes the proof.

We are now in the position to prove the second part of our main result in Theorem 1.1:

Proof of Theorem 1.1 ii. Let $\varphi: \mathbb{H}^{n} \rightarrow \mathbb{R}$ be an $H$-convex function with round $H$ sections. The previous arguments, together with Lemma 6.1 and Lemma 2 in [1], give that

$$
d_{\varphi}\left(\xi, \xi^{\prime}\right)=\inf \left\{s>0: \xi \in \mathbb{S}_{\varphi}^{\mathbb{H}^{n}}\left(\xi^{\prime}, s\right), \xi^{\prime} \in \mathbb{S}_{\varphi}^{\mathbb{H}^{n}}(\xi, s)\right\}
$$

is a quasi-distance in $\mathbb{H}^{n}$. Moreover, if $B_{\varphi}(\xi, r)$ denotes the $d_{\varphi}$-ball of center $\xi \in \mathbb{H}^{n}$ and radius $r>0$, we have that there exists $H$ which depends only on $K_{0}$ in (3.4) such that

$$
\mathbb{S}_{\varphi}^{\mathbb{H}^{n}}\left(\xi, \frac{r}{2 H}\right) \subset B_{\varphi}(\xi, r) \subset \mathbb{S}_{\varphi}^{\mathbb{H}^{n}}(\xi, r)
$$

The definition of $\mathbb{H}^{n}$-sections via subsequent constructions of $H$-sections makes hard its description in terms of functional inequalities. However, in the very simple case of the function $\varphi: \mathbb{H} \rightarrow \mathbb{R}$ defined by $\varphi(x, y, t)=x^{2}+y^{2}$, we are able to fully describe the set $\mathbb{S}_{\varphi}^{\mathbb{H}}(e, r)$ by providing explicitly the equation of its boundary. While in the Euclidean case the function $u(x)=\|x\|^{2}$, with $x \in \mathbb{R}^{n}$, gives rise to the sections $S_{u}\left(x_{0}, s\right)=B^{\mathbb{R}^{n}}\left(x_{0}, \sqrt{s}\right)$, i.e., the usual balls in $\mathbb{R}^{n}$, in the case of the first Heisenberg group $\mathbb{H}$ and with the mentioned function $\varphi$ we obtain $S_{\varphi}^{\mathbb{H}}\left(\xi_{0}, s\right)=\widetilde{B}\left(\xi_{0}, \sqrt{s}\right)$, and the family of $\mathbb{H}$-sections of $\varphi$ consists of the $\widetilde{B}$-balls in (5.2).

Example 6.1 Let us consider $\varphi: \mathbb{H} \rightarrow \mathbb{R}$ defined by $\varphi(x, y, t)=x^{2}+y^{2}$. This function is $\mathbb{R}^{3}$-convex, and hence $H$-convex. Since $\partial_{H} \varphi(x, y, t)=\{2(x, y)\}$, the horizontal section $S_{\varphi}^{H}\left(\xi_{0}, s\right)$ is given by

$$
S_{\varphi}^{H}\left(\xi_{0}, s\right)=\left\{\xi=(x, y, t) \in H_{\xi_{0}}:\left(x-x_{0}\right)^{2}+\left(y-y_{0}\right)^{2}<s\right\},
$$


for $\xi_{0}=\left(x_{0}, y_{0}, t_{0}\right)$ and $s>0$. Hence, for this particular $\varphi$, we have that

$$
S_{\varphi}^{H}\left(\xi_{0}, s\right)=B_{g}\left(\xi_{0}, \sqrt{s}\right) \cap H_{\xi_{0}},
$$

and, therefore,

$$
\overline{\mathbb{S}_{\varphi}^{\mathbb{H}}\left(\xi_{0}, s\right)}=\widetilde{B}\left(\xi_{0}, \sqrt{s}\right)
$$

Since, from the definition of $\mathbb{H}$-section, $\mathbb{S}_{\varphi}^{\mathbb{H}}\left(\xi_{0}, s\right)=\xi_{0} \circ \mathbb{S}_{\varphi}^{\mathbb{H}}(e, s)$, we will focus on the particular case $\xi_{0}=e$. We claim that, for every $r>0$,

$$
\begin{aligned}
\overline{\mathbb{S}_{\varphi}^{\mathbb{H}}(e, r)} & =\widetilde{B}(e, \sqrt{r}) \\
& =\left\{\xi=(x, y, t):|t| \leq \sqrt{3 r+2\|(x, y)\| \sqrt{r}-\|(x, y)\|^{2}}(\sqrt{r}+\|(x, y)\|)\right\} .
\end{aligned}
$$

Let us try to give the idea of its construction. Fix $r>0$. First of all, note that

- $\widetilde{B}(e, \sqrt{r})$ is radial with respect to the $t$-axis;

- $\widetilde{B}(e, \sqrt{r})$ is symmetric with respect to the $x y$-plane.

In particular, it is sufficient to identify the points of the set $\partial \widetilde{B}(e, \sqrt{r})$ in $\mathbb{H} \cap\{t \geq 0\}$. To this purpose, for every $\theta \in[-2 \pi / 3,0]$ let us consider the points

$$
\eta^{\theta}=(\sqrt{r}, 0,0) \circ(\sqrt{r} \cos \theta, \sqrt{r} \sin \theta, 0) \circ(\sqrt{r} \cos (2 \theta), \sqrt{r} \sin (2 \theta), 0),
$$

Trivially, $\eta^{0}=(3 \sqrt{r}, 0,0) \in \partial \widetilde{B}(e, \sqrt{r})$. Let us motivate our choice in (6.13). Let $v_{i} \in V_{1} \cong \mathbb{R}^{2}$, for $i=1,2,3$, and consider the point

$$
\eta=(x, y, t)=\exp \left(v_{1}\right) \circ \exp \left(v_{2}\right) \circ \exp \left(v_{3}\right)
$$

we have $(x, y)=v_{1}+v_{2}+v_{3}$, and $|t| / 4$ is equal to the area of the polygon $P=$ $\operatorname{co}\left\{(0,0), v_{1}, v_{1}+v_{2}, v_{1}+v_{2}+v_{3}\right\} \subset \mathbb{R}^{2}$, where "co" denotes the convex hull (for details on this application of Stokes' Theorem, see, for example, Section 2.3 in [12]). In order to construct $\partial \widetilde{B}(e, \sqrt{r}) \cap\{(x, y, t) \in \mathbb{H}: t \geq 0\}$ we restrict our attention to the points $\eta$ in (6.14) with the following features:

- $\left\|v_{i}\right\|=\sqrt{r}$

- the angles $\widehat{v_{1}, v_{2}}$ and $\widehat{v_{2}, v_{3}}$ are equal to $\theta$

(this choice will be explained later on). Due to the symmetries of $\widetilde{B}(e, \sqrt{r})$, we set

$$
v_{1}=\sqrt{r}(1,0), \quad v_{2}=\sqrt{r}(\cos \theta, \sin \theta), \quad v_{3}=\sqrt{r}(\cos (2 \theta), \sin (2 \theta)) .
$$

With this choice, from (6.13) one simply gets that

$$
\begin{aligned}
\eta^{\theta} & =(x(\theta), y(\theta), t(\theta)) \\
& =(\sqrt{r}(1+\cos \theta+\cos (2 \theta)), \sqrt{r}(\sin \theta+\sin (2 \theta)),-4 r \sin \theta(1+\cos \theta)) .
\end{aligned}
$$


Clearly, $t(\theta) \geq 0$ for $\theta \in[-\pi, 0]$. In the case $\theta=-2 \pi / 3, P$ turns out to be an equilateral triangle, and $\eta^{-2 \pi / 3}=(0,0, \sqrt{3} r)$; in the case $\theta \in[-\pi,-2 \pi / 3)$, we have that $\eta^{\theta}$ is an interior point of $\widetilde{B}(e, \sqrt{r})$. Therefore, we restrict our attention to the points $\eta^{\theta}$ as in (6.13). Simple computations give that, for $\theta \in[-2 \pi / 3,0]$,

$$
\begin{aligned}
& d(\theta):=\|(x(\theta), y(\theta))\|=\sqrt{r}(1+2 \cos \theta) \\
& t(\theta)=4 r \sqrt{1-\cos \theta}(1+\cos \theta) .
\end{aligned}
$$

Note that, if $\theta=-\pi / 3$, the function $t(\theta)$ reaches its maximum $3 \sqrt{3} r$ and, in this case, $d(-\pi / 3)=2 \sqrt{r}$. Consider the change of variable $z=\sqrt{r}(1+2 \cos \theta)$; due to the symmetry of $\widetilde{B}(e, \sqrt{r})$, we obtain that

$$
\left(z, 0, \sqrt{3 r+2 z \sqrt{r}-z^{2}}(\sqrt{r}+z)\right) \in \partial \widetilde{B}(e, \sqrt{r}), \quad \text { for } z \in[0,3 \sqrt{r}],
$$

and thus we get the expression in (6.12).

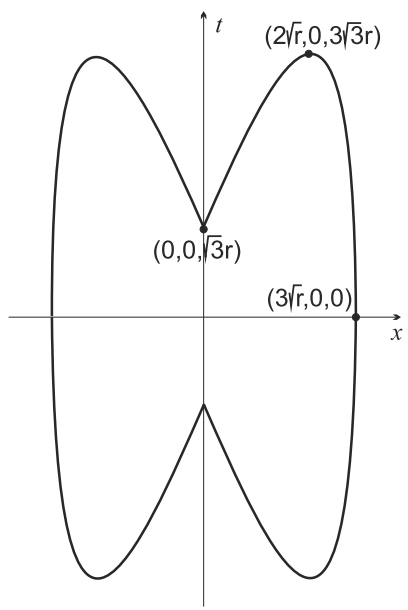

The profile in the plane $(x, 0, t)$ of the $\mathbb{H}-$ section $\mathbb{S}_{\varphi}^{\mathbb{H}}(e, r)$ of the function $\varphi: \mathbb{H} \rightarrow \mathbb{R}$ defined by $\varphi(x, y, t)=x^{2}+y^{2}$, for $r=1$.

Finally, let us explain briefly the restrictions imposed in (6.15) to obtain (6.12).

First, it is easy to see that, if in (6.14) we set $\left\|v_{i}\right\|=\sqrt{r}^{\prime}$, with $0<r^{\prime}<r$, we obtain that $\eta$ in (6.14) is in $\partial \widetilde{B}\left(e, \sqrt{r^{\prime}}\right) \subset \widetilde{B}(e, \sqrt{r})$; a similar argument holds for $\eta$ in (6.14), with the choice $\left\|v_{i}\right\|<\sqrt{r}$.

Secondly, let us motivate the restriction $\widehat{v_{1}, v_{2}}=\widehat{v_{2}, v_{3}}=\theta$ in (6.16). Fix $\theta \in$ $(-2 \pi / 3,0)$, consider $v_{i}$ as in (6.15) and the mentioned polygon $P$; using (6.16), the area of $P$ is exactly $-\sin \theta(1+\cos \theta)$. If one looks for the triplet of vectors $v_{i}$, with $\left\|v_{i}\right\|=\sqrt{r}$ for $i=1,2,3$, such that $v_{1}+v_{2}+v_{3}=(x(\theta), y(\theta))$ and such that the area of the associated polygon $P$ is the biggest one, then one obtains exactly the vectors $v_{i}$ in (6.15). This proves that $\eta^{\theta}$ belongs to the boundary of our $\mathbb{H}$-section. We leave the details and their tedious calculations to the interested reader. 


\section{Final Remarks and Open Questions}

Question 1 The assumption of the round $H$-sections property for an $H$-convex function $\varphi$ is a sufficient condition in order to guarantee that $\varphi$ satisfies the engulfing property $E\left(\mathbb{H}^{n}, K\right)$. It would be nice to weaken this assumption and prove that a function with the engulfing property $E(H, K)$ satisfies the engulfing property $E\left(\mathbb{H}^{n}, K\right)$.

Question 2 In [13] the authors study the engulfing property for convex functions in a generic Carnot group $\mathbf{G}$; as a matter of fact, in this more general framework, the related definition of G-sections (as in Definition 5.1) would be affected by the different geometry of the group $\mathbf{G}$, by the number of the steps and, especially, by the number of consecutive horizontal segments needed to connect any pair of points. Moreover, in a Carnot group with step greater than 2, a so-called horizontal line, i.e., a set $\{\xi \circ \exp s v\}_{s \in \mathbb{R}}$, is not a line in the Euclidean sense, as well as a horizontal plane is not a hyperplane in the Euclidean sense. This leads us to think that the $\mathbf{G}$-sections may have a very peculiar shape.

Question 3 By Theorem 3.3.10 in [19], the engulfing property for a convex function $\varphi: \mathbb{R}^{n} \rightarrow \mathbb{R}$ implies the existence of $C>0$ and $p \geq 1$ such that for every $0<r<$ $s \leq 1, x_{0} \in \mathbb{R}^{n}, t>0$, and $x \in S_{\varphi}\left(x_{0}, r t\right)$ we have the inclusion

$$
S_{\varphi}\left(x, C(s-r)^{p} t\right) \subset S_{\varphi}\left(x_{0}, s t\right) ;
$$

note that, under the assumptions above, the function $\varphi$ is differentiable (see [11]). Under any suitable version of the engulfing property in $\mathbb{H}^{n}$, can a similar inclusion be proved in $\mathbb{H}^{n}$ ?

Question 4 In [21] the authors prove, among other things, that the notion of round sections in Definition 2.1, controlled slope in (2.12), quasi uniform convexity, and quasiconformity are strictly related properties. To be precise, the next result holds (see Theorem 3.1 in [21]):

Theorem 7.1 Let $n \geq 2$, and let $u: \mathbb{R}^{n} \rightarrow \mathbb{R}$ be a convex function. The following are equivalent:

i. $u$ is quasiuniformly convex function, i.e. $u$ is not affine, $u \in W_{l o c}^{2, n}$ and there exists a constant $K \geq 1$ such that

$$
\left\|\nabla^{2} u(x)\right\|^{n} \leq K \operatorname{det} \nabla^{2} u(x), \quad \text { a.e. } x \in \mathbb{R}^{n} ;
$$

ii. $u$ is differentiable and $\nabla u: \mathbb{R}^{n} \rightarrow \mathbb{R}^{n}$ is quasiconformal, recalling that an injective map $F: \mathbb{R}^{n} \rightarrow \mathbb{R}^{n}$ is quasiconformal if $F \in W_{\text {loc }}^{1, n}$ and there exists a constant $K \geq 1$ such that

$$
\|\nabla F(x)\|^{n} \leq K \operatorname{det} \nabla F(x), \quad \text { a.e. } x \in \mathbb{R}^{n} ;
$$

iii. $u$ is differentiable, but not affine, and has controlled slope;

iv. $u$ has round sections. 
On the other hand, it is well known that the notion of quasiconformal map on $\mathbb{H}^{n}$ has been introduced and intensively studied (see for example [12]). In this paper we introduce the notion of $H$-controlled slope and round $H$-sections for an $H$-convex function but, at least to our knowledge, a horizontal notion of quasiuniform convexity for $H$-convex function does not exist in the literature. Our future aim will be to investigate a horizontal version of Theorem 7.1.

Funding Open access funding provided by Universitá degli Studi di Milano - Bicocca within the CRUICARE Agreement.

Open Access This article is licensed under a Creative Commons Attribution 4.0 International License, which permits use, sharing, adaptation, distribution and reproduction in any medium or format, as long as you give appropriate credit to the original author(s) and the source, provide a link to the Creative Commons licence, and indicate if changes were made. The images or other third party material in this article are included in the article's Creative Commons licence, unless indicated otherwise in a credit line to the material. If material is not included in the article's Creative Commons licence and your intended use is not permitted by statutory regulation or exceeds the permitted use, you will need to obtain permission directly from the copyright holder. To view a copy of this licence, visit http://creativecommons.org/licenses/by/4.0/.

\section{References}

1. Aimar, H., Forzani, L., Toledano, R.: Balls and quasi-metrics: a space of homogeneous type modeling the real analysis related to the Monge-Ampère equation. J. Fourier Anal. Appl. 4, 377-381 (1998)

2. Aliprantis, C.D., Border, K.: Infinite Dimensional Analysis. Springer, Berlin (2006)

3. Arena, G., Caruso, A., Monti, R.: Regularity properties of H-convex sets. J. Geom. Anal. 22, 583-602 (2012)

4. Balogh,Z.M., Calogero, A., Kristály, A.: Sharp comparison and Aleksandrov-type maximum principles in Heisenberg groups. J. Funct. Anal. 269, 2669-2708 (2015)

5. Balogh, Z.M., Rickly, M.: Regularity of convex functions on Heisenberg groups. Ann. Scuola Norm. Sup. Pisa Cl. Sci 2, 847-868 (2003)

6. Bonfiglioli, A., Lanconelli, E., Uguzzoni, F.: Stratified Lie Groups and Potential Theory for their Sub-Laplacians. Springer, New York (2007)

7. Caffarelli, L.A.: Some regularity properties of solutions of Monge-Ampère equation. Commun. Pure Appl. Math. 44, 965-969 (1991)

8. Caffarelli, L.A.: Boundary regularity of maps with convex potentials. Commun. Pure Appl. Math. 45, 1141-1151 (1992)

9. Calogero, A., Pini, R.: Horizontal normal map on the Heisenberg group. J. Nonlinear Convex Anal. 12(2), 287-307 (2011)

10. Calogero, A., Pini, R.: On Minty's theorem in the Heisenberg group. Nonlinear Anal. 104, 12-20 (2014)

11. Calogero, A., Pini, R.: The engulfing property from a convex analysis viewpoint. J. Optim. Theory Appl. 187, 408-420 (2020)

12. Capogna, L., Danielli, D., Pauls, S., Tyson, J.T.: An Introduction to the Heisenberg Group and the Sub-Riemannian Isoperimetric Problem. Birkhäuser, Basel (2007)

13. Capogna, L., Maldonado, D.: A note on the engulfing property and the $\Gamma^{1+\alpha}$-regularity of convex functions in Carnot groups. Proc. Am. Math. Soc. 134, 3191-3199 (2006)

14. Cruz-Uribe, D., Forzani, L., Maldonado, D.: The structure of increasing weights on the real line. Houston J. Math. 34(3), 951-983 (2008)

15. Danielli, D., Garofalo, N., Nhieu, D.M.: Notions of convexity in Carnot groups. Commun. Anal. Geom. 11, 263-341 (2003)

16. Folland, G.B., Stein, E.M.: Hardy Spaces on Homogeneous Groups. Princeton University Press, Princeton (1982)

17. Forzani, L., Maldonado, D.: On geometric characterizations for Monge-Ampère doubling measures. J. Math. Anal. Appl. 275(2), 721-732 (2002) 
18. Forzani, L., Maldonado, D.: Properties of the solution to the Monge-Ampère equation. Nonlinear Anal. 57, 815-829 (2004)

19. Gutiérrez, C.E.: The Monge-Ampère Equation. Birkhäuser, Boston, MA (2001)

20. Gutiérrez, C.E., Huang, Q.: Geometric properties of the sections of solutions to the Monge-Ampère equation. Trans. Am. Math. Soc. 352(9), 4381-4396 (2000)

21. Kovalev, L.V., Maldonado, D.: Mappings with convex potentials and the quasiconformal Jacobian problem. Illinois J. Math. 49(4), 1039-1060 (2005)

22. Kovalev, L.V., Maldonado, D., Wu, J.-M.: Doubling measures, monotonicity, and quasiconformality. Math. Z. 257, 525-545 (2007)

23. Magnani, V., Scienza, M.: Characterizations of differentiability for H-convex functions in stratified groups. Ann. Sc. Norm. Super. Pisa Cl. Sci., XIII:675-697, (2014)

24. Wang, X.J.: Some counterexamples to the regularity of Monge-Ampère equations. Proc. Am. Math. Soc. 123, 841-845 (1995)

Publisher's Note Springer Nature remains neutral with regard to jurisdictional claims in published maps and institutional affiliations. 